\title{
Malat1 regulates myogenic differentiation and muscle regeneration through modulating MyoD transcriptional activity
}

\author{
Xiaona Chen ${ }^{1}$, Liangqiang $\mathrm{He}^{2}$, Yu Zhao ${ }^{1}$, Yuying $\mathrm{Li}^{2}$, Suyang Zhang ${ }^{1}$, Kun $\mathrm{Sun}^{2}$, Karl So ${ }^{1}$, \\ Fengyuan Chen ${ }^{2}$, Liang Zhou', Leina Lu', Lijun Wang ${ }^{1}$, Xihua Zhu ${ }^{3}$, Xichen Bao ${ }^{3}$, Miguel A Esteban ${ }^{3}$, \\ Shinichi Nakagawa ${ }^{4}$, Kannanganattu V Prasanth ${ }^{5}$, Zhenguo $\mathrm{Wu}^{6}$, Hao Sun ${ }^{2, *}$, Huating Wang ${ }^{1, *}$ \\ ${ }^{1}$ Department of Orthopaedics and Traumatology, Li Ka Shing Institute of Health Sciences, Chinese University of Hong Kong, \\ Hong Kong, China; ${ }^{2}$ Department of Chemical Pathology, Li Ka Shing Institute of Health Sciences, Chinese University of Hong \\ Kong, Hong Kong, China; ${ }^{3}$ Genome Regulation Laboratory, Guangzhou Institutes of Biomedicine and Health, Chinese Academy \\ of Sciences, Guangzhou, China, ${ }^{4}$ RNA Biology Laboratory, RIKEN Advanced Research Institute, Wako, Japan; ${ }^{5}$ Department of \\ Cell and Developmental Biology, Chemical and Life Sciences Laboratory, University of Illinois at Urbana-Champaign, Urbana, \\ IL, USA; ${ }^{6}$ Division of Life Sciences, The Hong Kong University of Science and Technology, Hong Kong, China
}

\begin{abstract}
Malat1 is one of the most abundant long non-coding RNAs in various cell types; its exact cellular function is still a matter of intense investigation. In this study we characterized the function of Malat1 in skeletal muscle cells and muscle regeneration. Utilizing both in vitro and in vivo assays, we demonstrate that Malat1 has a role in regulating gene expression during myogenic differentiation of myoblast cells. Specifically, we found that knockdown of Malat1 accelerates the myogenic differentiation in cultured cells. Consistently, Malat1 knockout mice display enhanced muscle regeneration after injury and deletion of Malat1 in dystrophic mdx mice also improves the muscle regeneration. Mechanistically, in the proliferating myoblasts, Malat1 recruits Suv39h1 to MyoD-binding loci, causing trimethylation of histone 3 lysine 9 (H3K9me3), which suppresses the target gene expression. Upon differentiation, the pro-myogenic miR-181a is increased and targets the nuclear Malat1 transcripts for degradation through Ago2-dependent nuclear RNA-induced silencing complex machinery; the Malat1 decrease subsequently leads to the destabilization of Suv39h1/HP1ß/HDAC1-repressive complex and displacement by a Set7-containing activating complex, which allows MyoD trans-activation to occur. Together, our findings identify a regulatory axis of miR-181a-Malat1-MyoD/Suv39h1 in myogenesis and uncover a previously unknown molecular mechanism of Malat1 action in gene regulation.
\end{abstract}

Keywords: Malat1; miR-181; MyoD; myogenesis; Suv39h1

Cell Discovery (2017) 3, 17002; doi:10.1038/celldisc.2017.2; published online 14 March 2017

\section{Introduction}

Long non-coding RNAs (lncRNAs) are emerging as important regulators of gene expression in major biological processes in the nucleus or cytoplasm, having an impact on cell differentiation, stem cell function and

*Correspondence: Huating Wang

Tel: +852 3763 6047; Fax: +852 37636333 ;

E-mail: huating.wang@cuhk.edu.hk

or Hao Sun

Tel: +852 3763 6048;

E-mail: haosun@cuhk.edu.hk

Received 9 November 2016; accepted 29 December 2016 tissue development [1]. As more and more lncRNAs are being functionally characterized [2], a common theme is emerging for nuclear lncRNAs: they drive the formation of ribonucleic-protein complexes with numerous chromatin regulators and then target these enzymatic activities to appropriate locations in the genome, which in turn influence the gene regulation [3-6]. Malat1 (metastasis-associated lung adenocarcinoma transcript 1) is a lncRNA highly expressed in many tissues and regulated during tissue differentiation. Originally identified as a gene upregulated in metastatic non-small-cell lung cancer cells [7], human $M A L A T 1$ has recently been found overexpressed in 
many cancers $[8,9]$. It localizes to specific nuclear bodies called nuclear speckles [10] where it interacts with several serine- and arginine-rich (SR) family of splicing factors to regulate alternative splicing [11]. In cultured mouse hippocampal neurons, Malat modulates synapse formation by regulating the expression of genes involved in synaptogenesis [12]. Although emerging reports suggest Malat1 might influence gene expression at transcription level [9], the molecular interactions which allow for such function remain underexplored. RNA pull-down assay followed by mass spectrometry analysis conducted in HeLa cells identified that MALAT1 could bind to distinctive chromatin modifiers [13]. However, functional characterization of these interactions is still lacking.

In addition to interacting with proteins, emerging evidence indicates frequent association of MALAT1 with microRNAs. It has become an accepted concept that miRNA-lncRNA interaction is an existing phenomenon in cells [14]. The most well-known mode of the interaction is the competing endogenous model, that is, lncRNAs acting as sponges for miRNAs and competing for miRNAs' binding to bona fide mRNA targets [15]. However, recent reports support that interaction between $M A L A T 1$ and miRNA often leads to downregulation of MALAT1 abundancy [16-18]. Nevertheless, the underlying molecular mechanism remains unexplored, especially whether the canonical RISC machinery used for miRNA targeting in the cytoplasm is also utilized for the degradation of nuclear-residing MALAT1.

Myogenesis, which occurs in both postnatal growth and the regeneration of skeletal muscle after an injury, is a highly ordered process that can be subdivided into multiple steps. These steps include the activation of satellite cells (SCs or adult muscle stem cells) into committed myoblasts, followed by proliferation and differentiation of myoblasts, resulting in cell fusion to form myotubes $[19,20]$. Sequential activation of the muscle-specific transcriptional factors has a crucial role in regulating myogenesis [21]. Owing to the early discovery of the myogenic factors that act downstream, the myoblast differentiation has been extensively studied. It is a powerful system for investigating the biological functions of IncRNAs because it can be partially recapitulated in vitro using a $\mathrm{C} 2 \mathrm{C} 12$ murine myoblast cell line and because the transcriptional networks coordinating gene expressions are well investigated. Specification and differentiation of myoblasts into myotubes are driven by MyoD, Myf5, Myogenin and MRF4; upon induction by MyoD, Myogenin together with MyoD activates the transcription of muscle-specific genes such as myosin heavy chain (MyHC), alpha actin ( $\alpha$-actin) and troponin isoforms. Recent lines of work from our group [22-26] and others demonstrate that complex networks of transcription factors, chromatin regulators and microRNAs orchestrate tight control of myogenic differentiation through integrating intrinsic and extrinsic input into the gene expression program [21, 27, 28]. Emerging evidence from our group and others also demonstrates that lncRNAs are novel components of these regulatory networks $[15,29,30]$. Of particular interest, two recent studies $[31,32]$ investigated the function of Malat1 during myogenesis in $\mathrm{C} 2 \mathrm{C} 12$ showing promoting effect of Malat1 knockdown on $\mathrm{C} 2 \mathrm{C} 12$ differentiation. However, the underlying molecular mechanism needs to be further explored and the function of Malat1 in myogenesis also needs further clarification in a regeneration setting in vivo.

When associated to the consensus E-box sequence within muscle-specific gene promoters, MyoD, as a master regulator, can recruit multiple transcriptional regulators and interact with the basal transcriptional machinery to activate gene expression [33]. Chromatin immunoprecipitation-sequencing (ChIP-Seq) analysis has revealed that MyoD binds to a large number of genomic loci in proliferating myoblasts but is unable to activate their transcription [34], reinforcing the notion that MyoD activity must be precisely regulated to orchestrate the transcriptional program. One mechanism is through the association with Suv39h1, a histone H3 lysine 9 (H3-K9) methyltransferase. In the proliferating myoblasts, Suv39h1 is recruited to the MyoD-bound muscle gene loci to suppress their expression through its histone methyltransferase activity [35]; upon differentiation, Suv39h1 is replaced by Set7, which results in activation of the gene expression [36]. In addition to Suv39h1, MyoD also recruits HDACs and HP1 $\beta$ to target promoters, together forming a silencing complex to suppress gene expression by inducing a locally repressive chromatin structure [37]. Still, it is yet to answer the questions: how is Suv39h1 recruited to MyoD-bound loci? How is the complex stabilized in proliferating myoblasts and displaced upon differentiation?

To address the physiological function of Malat 1 in a living organism, three groups have independently generated Malat1 knockout (KO) mice using homologous recombination [38-40]. Unexpectedly, the Malat1-KO mice display no apparent abnormalities, suggesting that Malat1 is not essential in living mice maintained under normal conditions; its function probably becomes apparent only in specific cell types 
or under certain stress or pathological conditions. This also demonstrates that our understanding of the Malat 1 function in both physiological and pathological conditions is far from completion. In this project, we thus investigate the function of Malatl in skeletal muscle cells and muscle regeneration. Our findings reveal that Malat1 indeed has a critical role during myoblast differentiation. Knockdown of Malat1 accelerates the myogenic process. Functional elucidation uncovers a novel molecular mechanism of Malat1 regulating MyoD trans-activation through recruiting Suv39h1 to the MyoD-binding loci. In vivo, the injuryinduced muscle regeneration process is accelerated with genetic deletion of Malat1 owing to the enhanced myogenic capacity of SCs. Lastly, we also demonstrate that Malat1 transcript is directly targeted by miR-181a and degraded through Ago2-dependent nuclear RNAinduced silencing complex (nRISC) machinery occurring in the myoblast nucleus. Altogether, our results have identified a previously unknown molecular mechanism of Malat1 action.

\section{Results}

\section{Malat1 is dynamically expressed during skeletal muscle differentiation}

To investigate whether Malat1 is a functional molecule during skeletal muscle differentiation, we first examined its temporal and spatial expression patterns in several myogenesis systems in vitro and in vivo. When $\mathrm{C} 2 \mathrm{C} 12$ myoblasts were induced to differentiate by switching the cells from proliferation to serumdeprived differentiation medium (DM), according to the published high-throughput RNA-sequencing (RNA-seq) data [41], Malat1 expression was highly expressed in myoblasts and continuously upregulated in a course of $0,60,120$ and $168 \mathrm{~h}$ in DM (Figure 1a and $b$ ); this was confirmed by northern blot analysis: a strong signal was detected around $7 \mathrm{~kb}$ with the intensity increasing from 0 to 6 days in DM (Figure 1c). However, when taking a closer look at the earlier time points of differentiation $(<24 \mathrm{~h})$, Malat1 was markedly downregulated at DM $12 \mathrm{~h}(86 \%$ decrease) and $24 \mathrm{~h}(79 \%$ decrease), whereas the early-differentiation marker gene, Myogenin, was continuously induced (Figure 1d and Supplementary Figure S1a and b), suggesting that Malat1 may act as an anti-myogenic factor during the early differentiation of $\mathrm{C} 2 \mathrm{C} 12$ myoblasts. Consistently, during the differentiation of freshly isolated SCs, Malat1 was also remarkably decreased (Figure 1e). To further examine its expression dynamics during myogenesis in vivo, we employed a widely used muscle regeneration model in which the intramuscular injection of cardiotoxin results in muscle injury and in turn induces muscle regeneration $[29,30]$. Malat1 was found to be highly induced during the early regeneration stage when SCs became activated, proliferated and started to differentiate, but gradually downregulated later on when the newly formed fibers matured and regeneration completed in $\sim 9$ days (Figure 1f). Consistently, high level of Malat1 was observed in limb muscles of newborn mice (aged 3 days to 3 weeks), which displayed active myogenesis, but the level of Malat1 was decreased as the neonatal myogenesis ceased after $\sim 3$ weeks (Figure $1 \mathrm{~g}$ ). Moreover, when compared with normal muscles from wild-type (WT) mice, a higher level of Malat1 was detected in dystrophic muscles from young mdx mice, a mouse model of Duchenne muscular dystrophy at 3, 5 or 6 weeks of age, which were featured by a pathologically active degeneration-regeneration process (Supplementary Figure S1d). The above results suggested that Malat1 is associated with active myogenesis processes in vitro and in vivo.

To gain more functional insights, the subcellular localization of Malat1 was examined using RNA fluorescence in situ hybridization (FISH). In line with the previous reports showing that Malat1 is located in the nuclear speckles in many cell types [10, 11], Malat1 was found exclusively in the nucleus of $\mathrm{C} 2 \mathrm{C} 12$ myoblast (Figure 1h) and myotube (Supplementary Figure $\mathrm{S} 1 \mathrm{~b}$ ); as a positive control, the nuclear transcript snRNA $U 1$ was also found restrictively in the nucleus (Figure 1h). A high magnification of the FISH image revealed its focal distribution pattern (Supplementary Figure S1c), resembling the staining pattern of Srsf2, a marker for nuclear speckle, suggesting that a large portion of Malat1 may localize in the nuclear speckles in $\mathrm{C} 2 \mathrm{C} 12$ cells as in many other cell types [42]. By cellular fractionation assay, Malat1 transcripts were highly enriched in the insoluble fraction of nuclei where the chromatin-binding lncRNA Xist was also detected; $G A P D H$ transcripts, on the other hand, were mainly found in the cytoplasmic extracts (Figure 1i).

\section{Malat1 represses myoblast differentiation}

The early downregulation of Malatl expression during myoblast differentiation suggested that it might be an anti-myogenic factor in the initial stage of differentiation. To test this notion, we generated a C2C12 cell line stably expressing a short hairpin RNA (shRNA) against Malat1. Knockdown of Malat1 (Figure 2a, 53\% knockdown efficiency, and Figure 2b) significantly enhanced $\mathrm{C} 2 \mathrm{C} 12$ differentiation as 
a

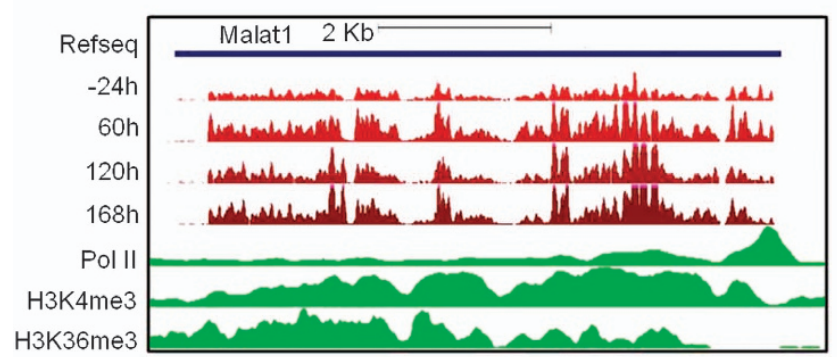

d
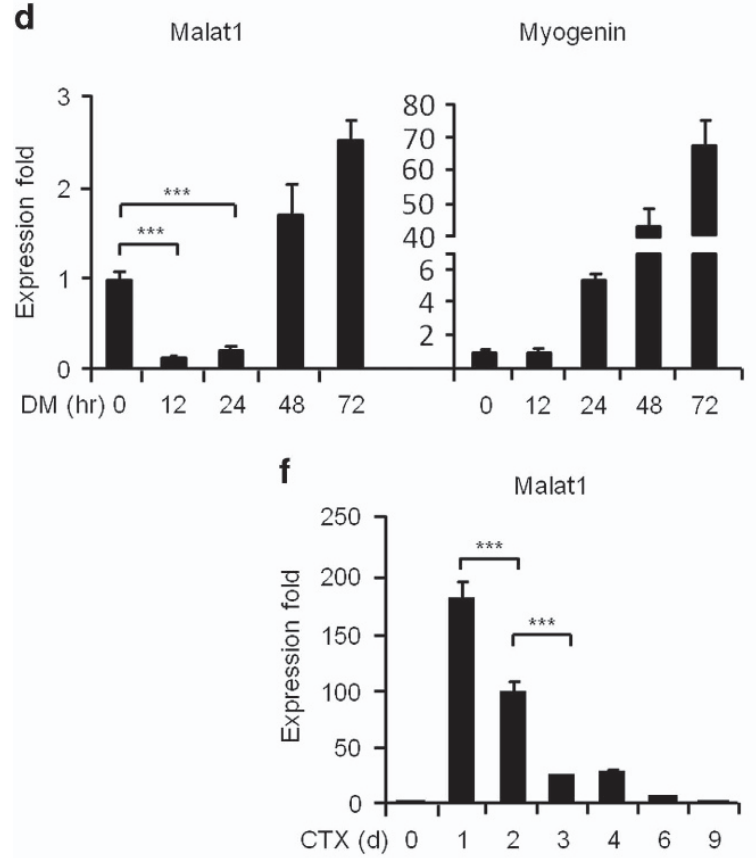

h

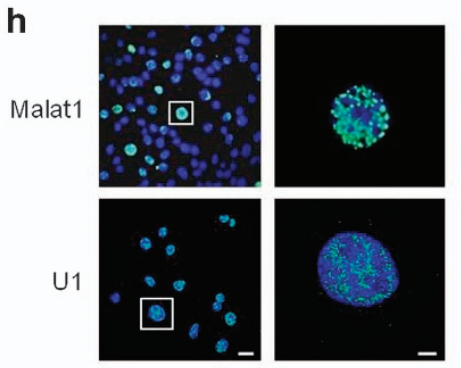

b
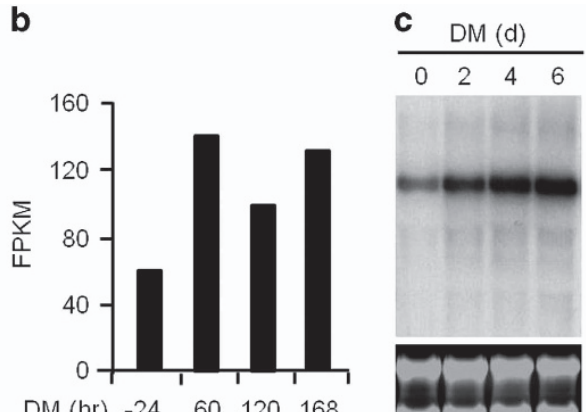

DM (hr) $-24 \quad 60 \quad 120 \quad 168$

e

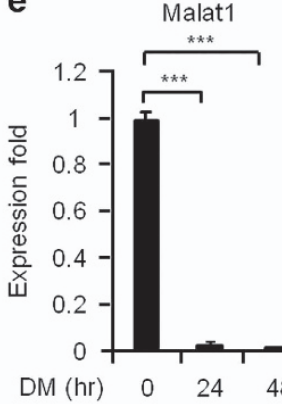

Myogenin

g

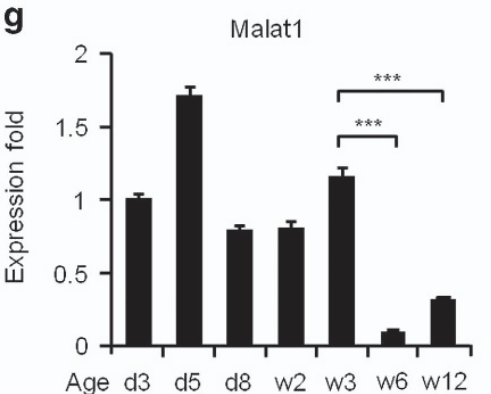

i

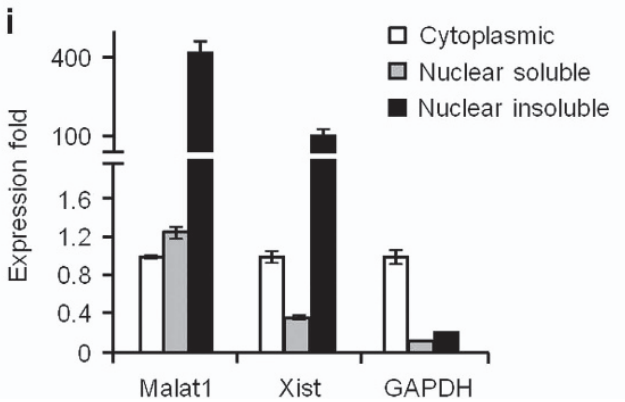

Figure 1 Malat1 is associated with skeletal myogenesis. (a) Genomic snapshot of mouse Malat1 generated in Refseq (blue), RNA-seq (red, from C2C12 cells in DM for -24, 60, 120 and 168 h), Pol II, H3K4me3 and H3K36me3 ChIP-seq (green) tracks from $\mathrm{C} 2 \mathrm{C} 12$ cells in growing medium. (b) The Fragments Per Kilobase of transcripts per Million mapped reads (FPKM) values of Malat1 from the above RNA-seq data. (c) The expression of Malat1 at the indicated time points in DM was detected by northern blotting. 18S and 28S rRNAs are used as loading controls. The size of the ladder was shown on the right. (d) The expression of Malat1 and Myogenin during C2C12 differentiation was examined by qRT-PCR. (e) The expression of Malat1 and Myogenin in early-differentiating satellite cells freshly isolated from mouse limb muscles. (f) The expression of Malat1 during CTX-induced regeneration at the indicated days post-CTX injection; (g) in muscles of postnatal mice at the indicated age (week); (h) visualization of Malat1 or U1 transcripts in $\mathrm{C} 2 \mathrm{C} 12$ myoblasts by RNA FISH. The images on the right are the boxed regions in a higher magnification ( $\times 1000$ ). Left panel scale bar $=20 \mu \mathrm{m}$. Right panel scale bar $=5 \mu \mathrm{m}$. (i) The expression of Malat1, Xist and $\mathrm{GAPDH}$ in three fractions extracted from $\mathrm{C} 2 \mathrm{C} 12$ myoblasts: cytoplasmic, nuclear-soluble or nuclear-insoluble fraction. All PCR data were normalized to GAPDH mRNA and represent the average of three independent experiments \pm s.d. ${ }^{* * *} P<0.001$. 

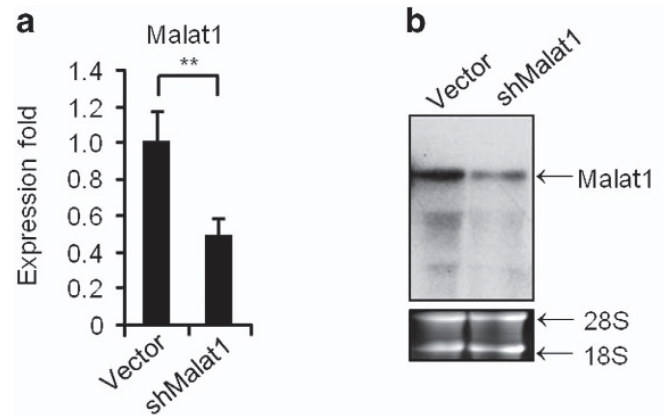

C
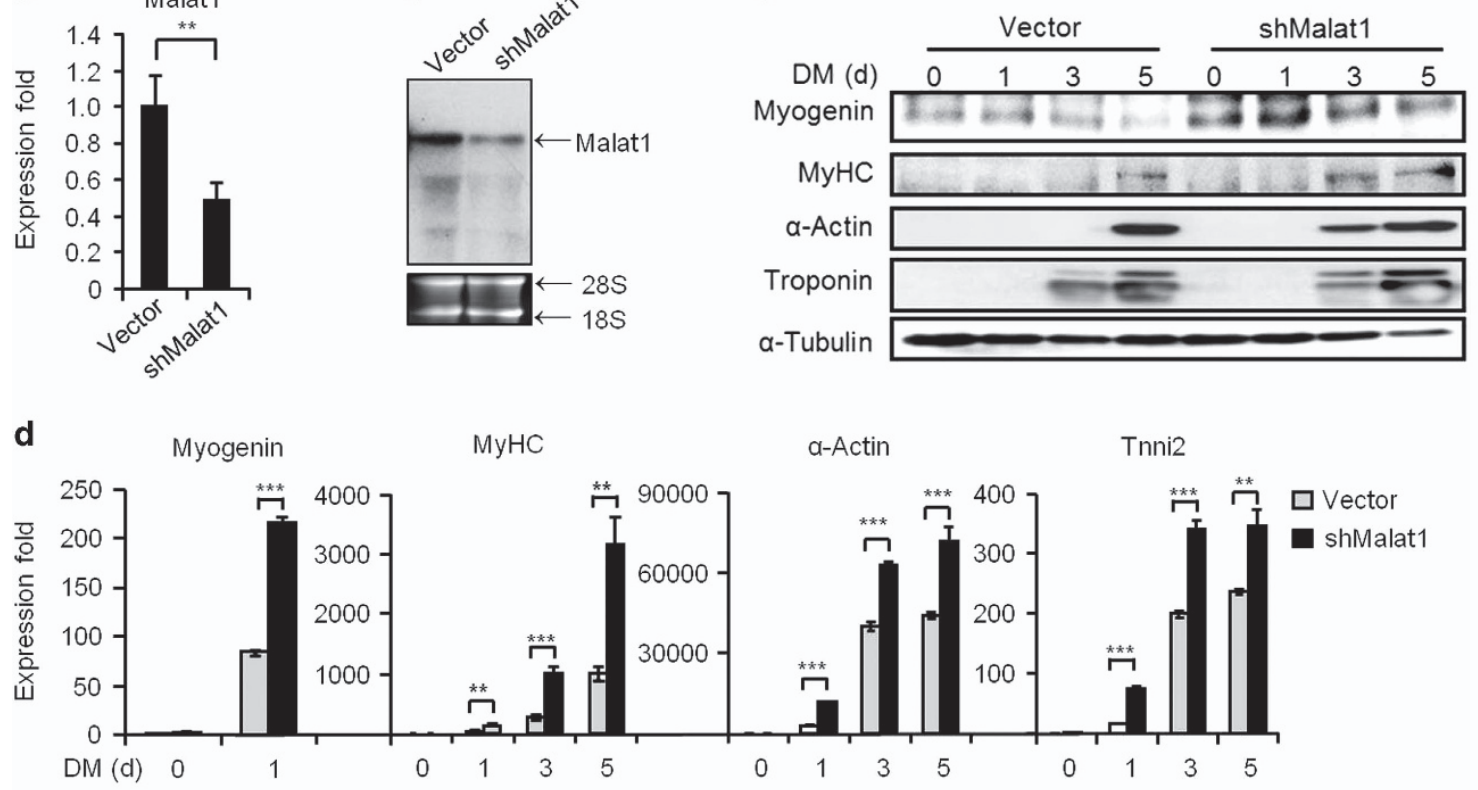

e

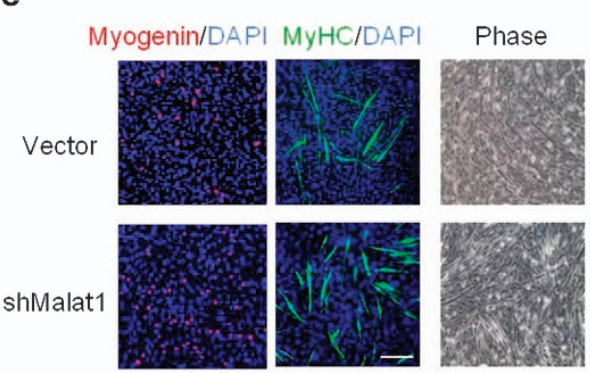

f
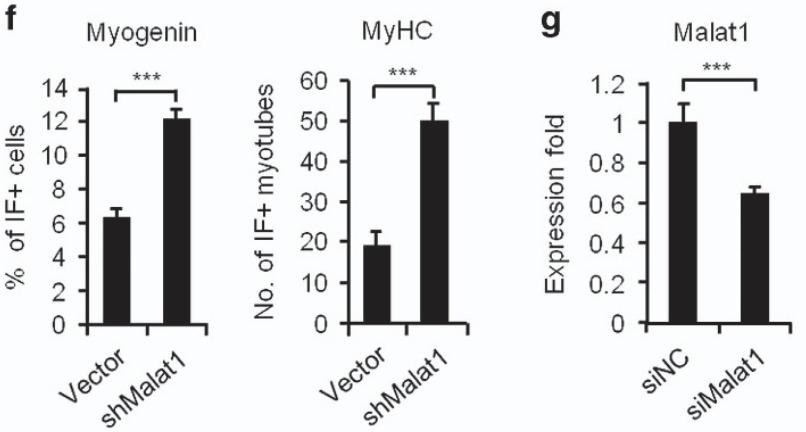

n

i

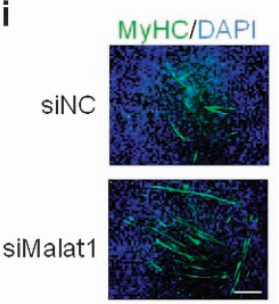

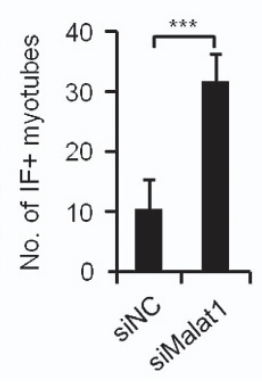

Figure 2 Malat1 functions to repress myoblast differentiation. (a, b) The knockdown of Malat1 in C2C12 cells by a stably expressed shRNA oligo was confirmed by qRT-PCR (a) and northern blotting in proliferating myoblasts (b). (c) The knockdown of Malat1 increased the levels of the indicated myogenic genes, Myogenin, MyHC, $\alpha$-Actin and Troponin at protein level during a 5-day differentiation course. (d) The knockdown of Malat1 increased the above myogenic genes at mRNA levels. (e) The above cells were visualized on day 2 in DM (phase images). IF staining for Myogenin or MyHC was performed on day 1 and day 3 in DM, respectively. (f) The number of positively stained cells was counted from at least 10 fields. (g) The knockdown of Malat1 by a siRNA oligo was confirmed by qRT-PCR on day 2 post transfection. (h) The above knockdown increased the levels of the indicated myogenic genes $\alpha$-Actin and Troponin at the protein level during a 4-day differentiation course. (i) The above siMalat1 transfection also increased the number of MyHC-positive cells by IF staining on day 2 in DM. (j) The knockdown of Malat1 in $\mathrm{C} 2 \mathrm{C} 12$ cells increased the luciferase activities of the indicated myogenic reporters on day 2 in DM. RLU, relative luciferase unit. All PCR data were normalized to GAPDH mRNA and represent the average of three independent experiments \pm s.d. All luciferase data were normalized to Renilla luciferase activities and represent the average of three independent experiments \pm s.d. ${ }^{*} P<0.05,{ }^{* *} P<0.01,{ }^{* * *} P<0.001$. 
assessed by the examination of several myogenic markers, Myogenin, MyHC, $\alpha$-Actin and Troponin, at both protein (Figure 2c) and RNA (Figure 2d) levels during a differentiation course of 5 days. The results were also strengthened by immunofluorescence (IF) staining for Myogenin and MyHC proteins in differentiating myotubes on day 1 and 3 , respectively (Figure 2e); the number of positively stained myotubes a

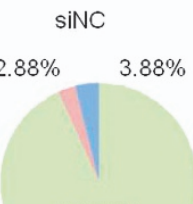

$93.24 \%$
siMalat1

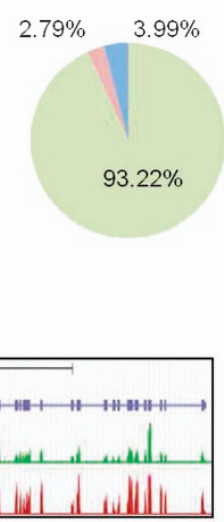

d

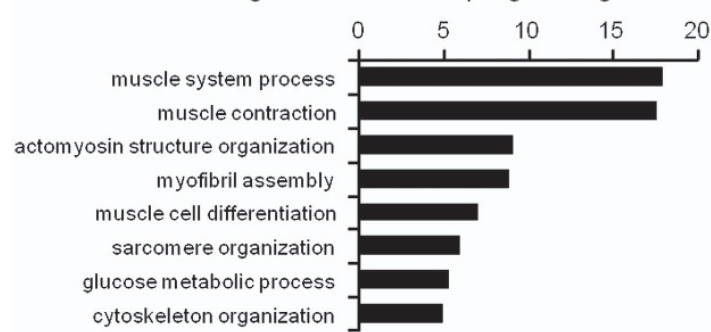

C

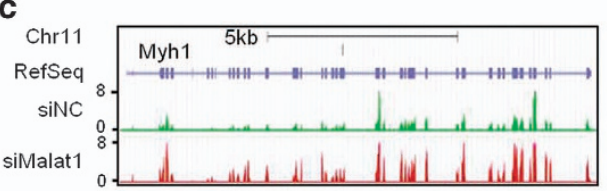

Exonic

E Intronic

= Intergenic

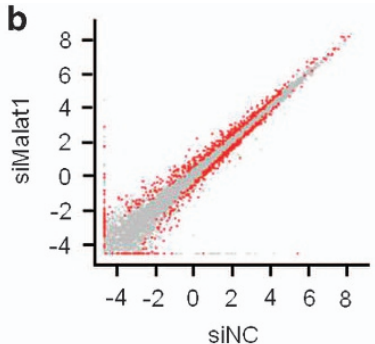

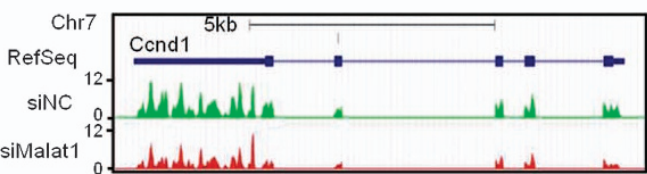

GO categories enriched in down-regulated genes

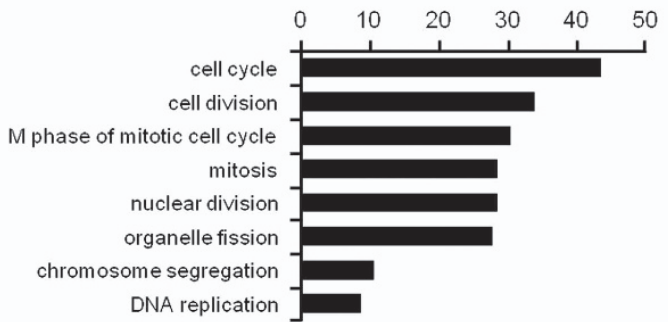

e

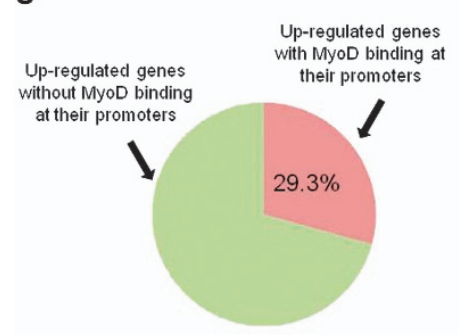

f

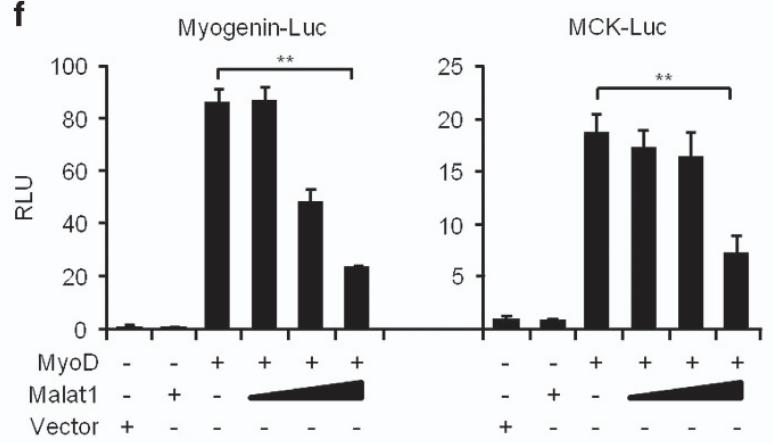

h

g Myogenin-Luc MCK-Luc

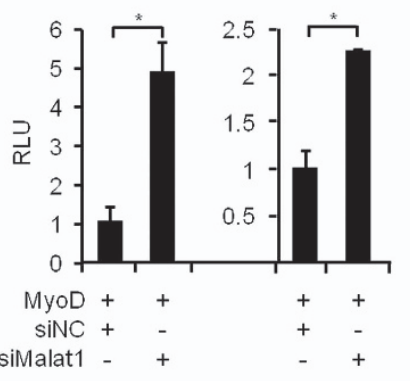

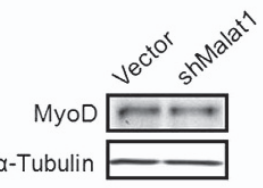

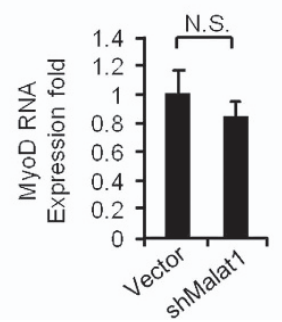

i

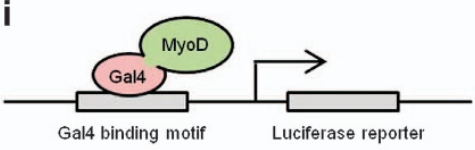

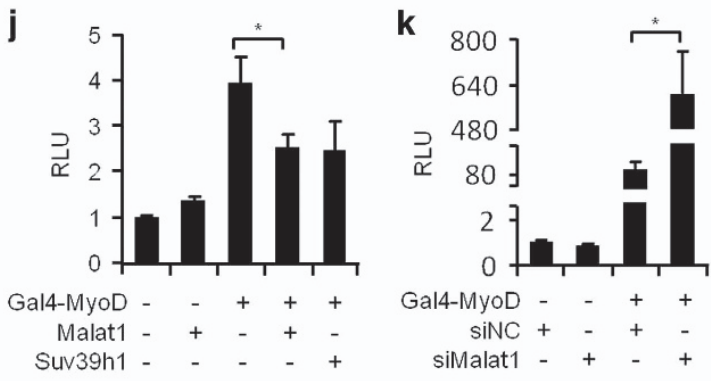

Cell Discovery | www.nature.com/celldisc 
was increased by shMalat1 treatment by 1.9- and 2.7fold, respectively (Figure 2f). To confirm the above results, transient transfection of a short interfering RNA (siRNA) oligo against Malat1 to $\mathrm{C} 2 \mathrm{C} 12$ cells was performed (Figure 2g, 36\% knockdown efficiency), and the same accelerating effect was observed by assaying for expression of the myogenic markers (Figure $2 \mathrm{~h}$ and i). Furthermore, transfection of a myogenic luciferase reporter together with siMalat1 oligos significantly increased the reporter activities as compared with negative control oligos (Figure 2j, Myog-Luc, 4.3-fold; Tnni2-Luc, 4.2-fold; Muscle creatine kinase, MCK-Luc, 3.4-fold; MyHC-Luc, 4.5-fold). Owing to its large size and high abundance, Malat1 is notorious for the difficulty in knocking down; we managed to find a second siRNA oligo targeting a different region of Malatl, which also led to a similar pro-myogenic phenotype (Supplementary Figure S2h-j). Conversely, increase in the Malat 1 level by directly transfecting in vitro-transcribed Malatl transcripts suppressed the C2C12 differentiation (Supplementary Figure S2a-c). In addition, Malat1 appeared to promote $\mathrm{C} 2 \mathrm{C} 12$ myoblast proliferation as its knockdown inhibited cell proliferation as assessed by viable cell counting (Supplementary Figure S2d), MTT assay (Supplementary Figure S2e) and 5-bromo-2'-deoxyuridine (BrdU) staining (Supplementary Figure S2f, 10\% decrease by siMalat1), whereas overexpression of Malat1 accelerated myoblast proliferation (Supplementary Figure S2g).
Malat1 represses the transcriptional activity of $M y o D$

To gain insights into molecular mechanisms of how Malat1 regulates the myogenic differentiation, we conducted an RNA-seq analysis to globally characterize the transcriptomic changes affected by Malat1 . Polyadenylated RNAs were extracted from $\mathrm{C} 2 \mathrm{C} 12$ cells transfected with siMalat or siNC oligos and subjected to RNA-seq analysis (Figure $3 \mathrm{a}$ and Supplementary Figure S3a and b). As a result, a total of 427 genes were upregulated, whereas 403 were downregulated (Figure $3 \mathrm{~b}$ and $\mathrm{c}$ and Supplementary Table S1), indicating that Malat1 knockdown induced a global transcriptomic change in the muscle cell. Subsequent GO analysis revealed that the upregulated genes were enriched for muscle-related terms (Figure 3d, left). The downregulated genes, on the other hand, were enriched for cell cycle-related terms (Figure 3d, right). This was consistent with the enhanced differentiation and inhibited proliferation phenotype that we observed in Figure 2. Many of the upregulated genes were muscle genes known to be regulated by the muscle master transcription factor, MyoD, leading us to suspect that Malatl's function may be associated with MyoD regulation. Indeed, by exploring MyoD ChIP-Seq data from $\mathrm{C} 2 \mathrm{C} 12$ cells [34], we found that almost $30 \%$ of above upregulated genes had MyoD binding at their promoters (Figure 3e).

To further test Malat1 regulation of MyoD activity, C3H/10T1/2 (10T1/2) fibroblasts were co-transfected

Figure 3 Malat1 modulates the transcriptional activity of MyoD. (a) RNAs with PolyA tails (PolyA RNAs) were isolated from C2C12 cells transfected with siNC or siMalat1 oligos and differentiated for 2 days and subjected to RNA-seq for transcriptomic analysis. The normalized fragment density was calculated by counting the fragments per kilobase of genomic regions of interests (exonic, intronic and intergenic) per million mapped reads (FPKM). (b) Differentially expressed genes between siNC ( $x$ axis) and siMalat1 ( $y$ axis) cells were determined from the above RNA-seq data and shown as red dots in the scatter plot. Gray dots represent genes unaffected by siMalat1 treatment. (c) Genomic snapshots showing an example of significantly up- (Myh1, left) or down- (Ccnd1, right) regulated genes by siMalat1 treatment. The $y$ axis is the normalized signal density. (d) GO analysis of genes that were up- or downregulated in siMalat1 cells. The $y$ axis shows GO terms and the $x$ axis shows statistical significance (that is, $-\log (p)$, multicorrected $P$-values) for the top eight enriched terms. (e) A high percentage (29.3\%; in red color) of the above upregulated genes contain a MyoD-binding site at their promoter regions based on the analysis of MyoD ChIP-seq data from C2C12 cells. (f) 10T1/2 cells were co-transfected with a Myogenin or MCK luciferase reporter, a MyoD expression plasmid $(0.5 \mu \mathrm{g})$ and a Malat1 expression plasmid $(0,0.125,0.25 \mathrm{or} 0.5 \mu \mathrm{g})$. Luciferase activities were measured after differentiating the cells for $48 \mathrm{~h}$. (g) $10 \mathrm{~T} 1 / 2$ cells were transfected with the indicated reporter, the MyoD plasmid and the siRNAs against Malat1 or negative control ( $\mathrm{iNC}$ ) oligos. Luciferase activities were measured after differentiating the cells for $48 \mathrm{~h}$. (h) Knockdown of Malat1 did not affect the expression of MyoD at protein (upper, western blotting) or RNA (bottom, qRT-PCR) level. (i) Schematic illustration of the Gal4-MyoD reporter system. A Gal4-binding motif is fused to the luciferase gene; the expression of a fusion protein with Gal4-binding domain fused with MyoD (Gal4-MyoD) will lead to its binding to the Gal4 motif and activate the luciferase reporter. (j) 10T1/2 cells were co-transfected with the above Gal4 luciferase reporter, the Malat1 expression vector ( $0.5 \mu \mathrm{g})$ or a Suv39h1 expression plasmid in the presence of the vector expressing Gal4-MyoD fusion protein. Luciferase activities were measured after differentiating the cells for $48 \mathrm{~h}$. (k) 10T1/2 cells were co-transfected with the Gal4 luciferase reporter, siMalat1 or siNC oligos and the Gal4-MyoD vector. Luciferase activities were measured after differentiating the cells for $48 \mathrm{~h}$. All PCR data were normalized to GAPDH mRNA and represent the average of three independent experiments \pm s.d. All luciferase data were normalized to Renilla luciferase activities and represent the average of three independent experiments \pm s.d. ${ }^{*} P<0.05,{ }^{* *} P<0.01$. 
with a MyoD expression plasmid and a MyoDresponsive luciferase reporter (Myogenin-Luc or MCK-Luc) in the presence of an increasing amount
$(0,0.125,0.25$ or $0.5 \mu \mathrm{g})$ of Malat 1 -encoding plasmid. The results from luciferase assays demonstrated that Malat1 inhibited the MyoD trans-activation of
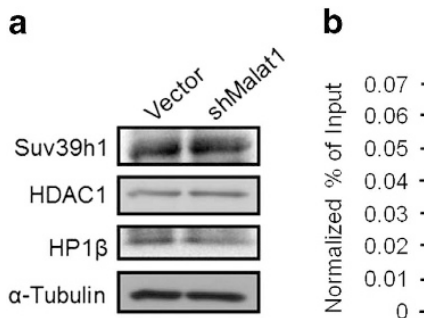

Malat1
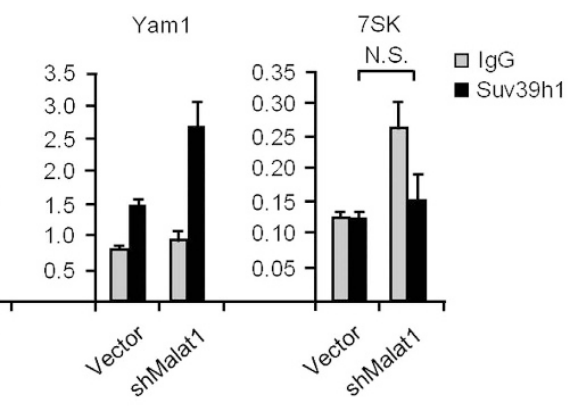

C

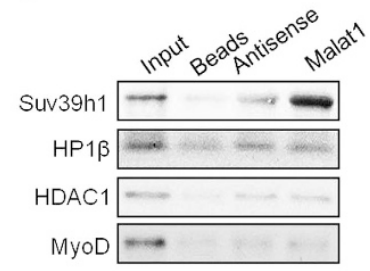

d

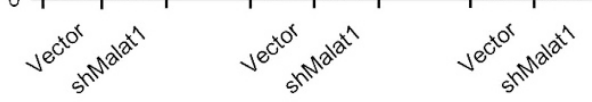

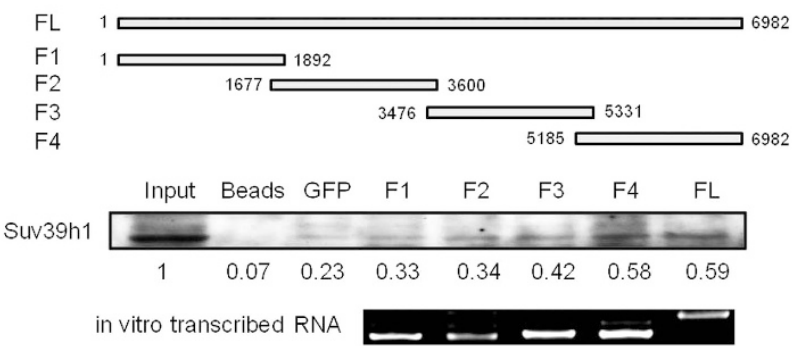

e
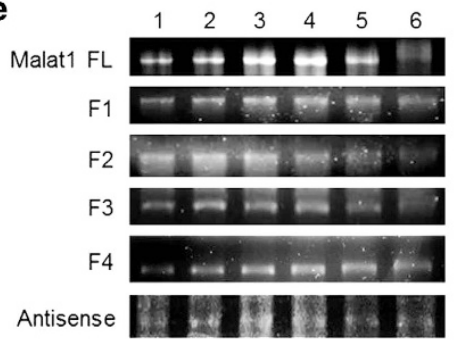

Malat1 RNA ++
GST GST-Suv39h1

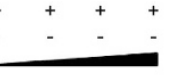

f

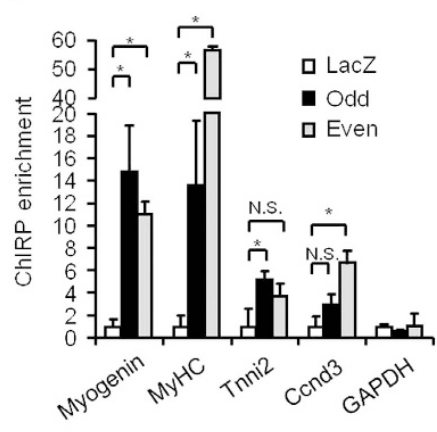

g

h

Myogenin MCK Tnni2

\begin{tabular}{lll}
$\mathrm{MyHC}$ & $\mathrm{Cond3}$ & $\mathrm{p} 21$ \\
2 & 2.5 \\
\hline
\end{tabular}

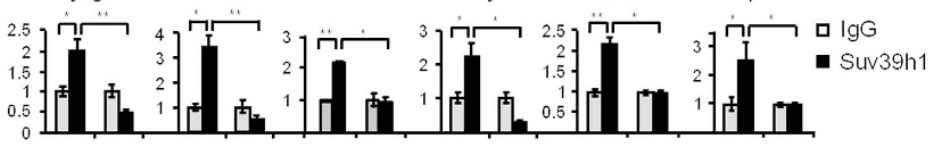

i
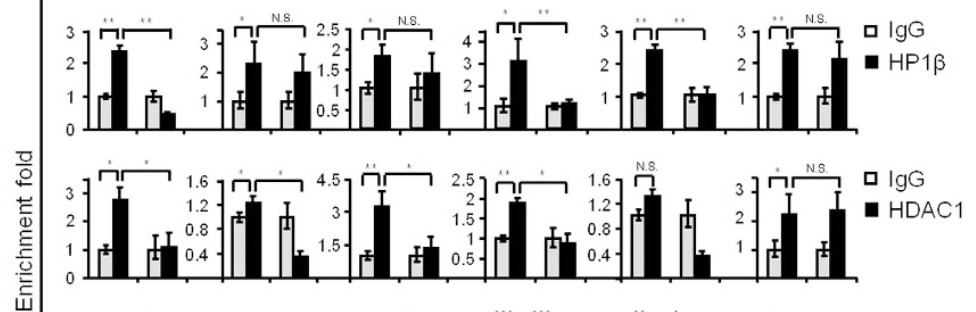

j

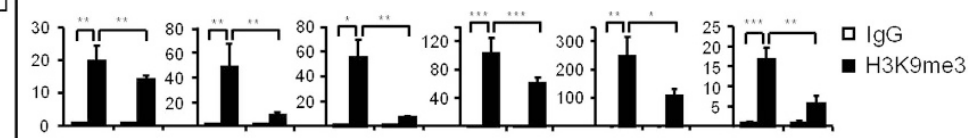

k

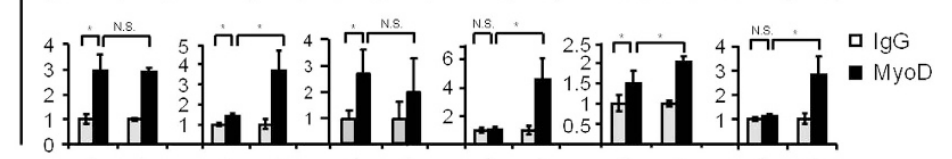

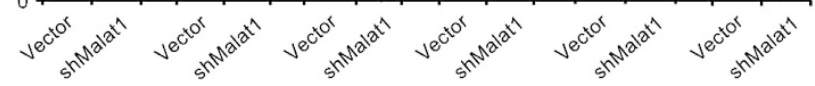

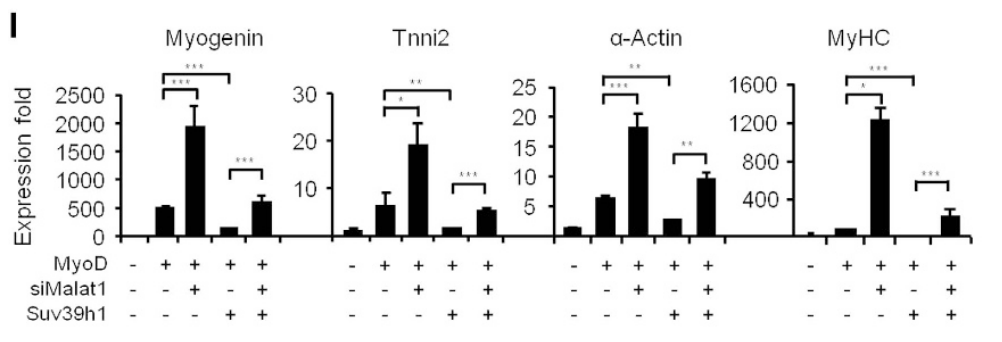

m Myog-Luc 4RE-Luc

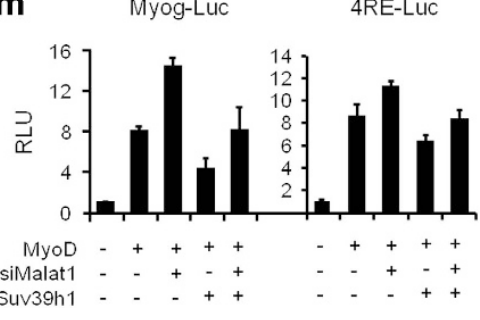

Cell Discovery | www.nature.com/celldisc 
these reporters in a dose-dependent manner (Figure 3f; Supplementary Figure S3c and e). On the contrary, transfection of siMalatl oligos led to enhanced activation of the myogenic reporters by MyoD (Figure 3g; Supplementary Figure S3d and f). Altogether, the above data suggested that Malat1 regulates myogenic differentiation through modulating MyoD activity. Interestingly, shMalat1 knockdown did not affect the expression of MyoD itself (Figure 3h). To further examine whether Malat1 regulates the trans-activation function of MyoD, we employed a Gal4 luciferase reporter system. As illustrated in Figure 3i, a Gal4-binding motif is fused to the luciferase gene; the expression of a fusion protein with Gal4 DNA-binding domain fused with MyoD (Gal4-MyoD) will lead to its binding to the Gal4 motif and activate the luciferase reporter. 10T1/2 cells were co-transfected with the above Gal4 luciferase reporter and the Gal4-MyoD fusion plasmid in the presence or absence of the Malat1-expressing plasmid. Trans-activation of the reporter was found to be inhibited by Malat1 expression (Figure $3 \mathrm{j}$ and Supplementary Figure S3g). Ectopic expression of Suv39h1, as previously shown [35], also inhibited the MyoD trans-activation (Figure 3j). Conversely, cotransfection of siMalatl oligos enhanced the MyoD trans-activation in the assay (Figure 3k and Supplementary Figure S3h-j). These results suggested that Malat1 could regulate the trans-activating ability of MyoD.
Malat1 recruits Suv39h1 to the MyoD-binding loci

To further investigate how Malat1 inhibits the trans-activating ability of MyoD, we thought of the possibility that Malat1 could recruit a repressive cofactor to the MyoD-binding loci. As Malat1 had been shown to interact with Set domain-containing proteins [13], we hypothesized that Malat1 might interact with Suv39h1 and recruit it to MyoD. To test this notion, we need to first exclude the possibility that Malat1 modulates Suv39h1 activity through regulating its expression. Indeed, the levels of Suv39h1 and its partners in the repressive complex, HP1 $\beta$, and HDAC1, were not affected by shMalat 1 treatment in C2C12 myoblasts (Figure 4a). Next, using crosslinked RNA immunoprecipitation (RIP) assay, antibodies against Suv39h1 protein retrieved a significant amount of endogenous Malat1 transcripts from $\mathrm{C} 2 \mathrm{C} 12$ myoblasts (3.2-fold enrichment compared with IgG pull-down; Figure 4b), whereas Yaml [29] and 7SK [43] were not retrieved, indicating a putative interaction between Suv39h1 protein and Malat1 transcripts. To strengthen this finding, we then further performed RNA pull-down assay from native non-crosslinked cell lysates using biotinylated in vitro-transcribed full-length (FL) Malat1 transcripts. Consistently, Malat1 transcripts pulled down a substantial amount of endogenous Suv39h1 protein; however, no HP1 $\beta$, HDAC1 or MyoD protein was retrieved (Figure 4c), suggesting a direct physical interaction between Suv39h1 and Malat1. As negative controls, beads

Figure 4 Malat1 interacts and recruits Suv39h1 to MyoD-binding loci. (a) The expression levels of Suv39h1, HDAC1 and Hp1 $\beta$ proteins in Vector or shMalat1 $\mathrm{C} 2 \mathrm{C} 12$ cells were determined by western blotting in proliferating myoblasts. $\alpha$-Tubulin was used as a normalization control. (b) Nuclear extracts from the above cells were immunoprecipitated by lgG or an antibody against Suv39h1. The retrieved RNAs were detected by qRT-PCR. (c) In vitro-transcribed biotinylated Malat1 FL transcripts were used to pull down its binding proteins. The indicated proteins were detected by western blotting. Beads only or antisense Malat1 transcripts were used as negative controls. (d) Upper: schematic illustration of the Malat1 FL or fragments (F1, F2, F3 or F4) that were used in the RNA pull-down assays for mapping the functional domain. Beads only or GFP transcripts were used as negative controls. Middle: the retrieved Suv39h1 proteins were detected by western blotting. The numbers below indicate the quantification of the intensity of each band. Lower: the in vitro generated transcripts were visualized on an agarose gel. (e) EMSA assays were performed to detect the association between Malat1 FL or various fragments with purified GST-Suv39h1 protein (for $200 \mathrm{fmol}$ Malat1 FL RNA, 100, 400, 800 or 1600 nM GST-Suv39h1 were used. For 400 fmol F1-F4 fragments, 800, 1600,2400 or 3200 nm GST-Suv39h1 protein were used). Antisense Malat1 transcript was used as an unrelated negative control for RNA. GST only protein (3 $200 \mathrm{nm)} \mathrm{was} \mathrm{used} \mathrm{as} \mathrm{a} \mathrm{negative} \mathrm{control} \mathrm{for} \mathrm{GST-Suv39h1} \mathrm{protein.} \mathrm{A} \mathrm{shifted} \mathrm{band}$ (lanes 5 and 6) ran higher than the control bands (lanes 1 and 2) and also displayed lower intensity. (f) Malat1 ChIRP with both even and odd antisense oligos retrieved a significant amount of genomic DNAs corresponding to the promoter regions of Myogenin, MyHC, Tnni2 and Ccnd3 genes but not GAPDH locus. LacZ ChIRP retrieved no signal. (g-k) ChIP-PCR analysis of Suv39h1, HP1 $\beta$, HDAC1, H3K9me3 and MyoD enrichment on the promoter or enhancer of Myogenin, MCK, MyHC, Troponin, Ccnd3 and p21 loci in Vector or shMalat1 expressing C2C12 cells. The enrichment fold was calculated as a fraction of DNA present in the input samples. (I) 10T1/2 cells were transfected with the indicated combination of plasmids and siMalat1 oligos. The expression of the myogenic marker genes was analyzed by qRT-PCR. (m) 10T1/2 cells were co-transfected with a Myogenin or 4RE luciferase reporter, the indicated expression plasmids and siMalat1 oligos. Luciferase activities were measured after differentiating the cells for $48 \mathrm{~h}$. All PCR data were normalized to GAPDH mRNA and represent the average of three independent experiments \pm s.d. All luciferase data were normalized to Renilla luciferase activities and represent the average of three independent experiments \pm s.d. ${ }^{\star} P<0.05,{ }^{* \star} P<0.01,{ }^{* \star *} P<0.001$. 
alone, green fluorescent protein (GFP) transcripts or antisense Malat1 transcripts did not retrieve Suv39h1 protein either (Figure 4c). To further map the binding domain, a series of deletion mutants of Malat1 was tested for the binding with Suv39h1. Interestingly, the 3' domain (5 185-6982 nt, F4) alone retrieved almost the equal amount of Suv39h1 as the FL transcript; fragments F2 and F3 also exhibited some binding activity, whereas fragment F1 had very low binding with Suv39h1 (Figure 4d). Similar results were obtained when a Flag-tagged Suv39h1 was expressed in $\mathrm{C} 2 \mathrm{C} 12$ cells and used in the assay (Supplementary Figure S4a). To further confirm the direct binding between Malat1 and Suv39h1, we performed in vitro RNA-protein electrophoretic mobility shift assay (EMSA). Purified GST (glutathione $S$-transferase)Suv39h1 protein and in vitro-transcribed Malat1 full length or fragments were incubated and analyzed for their binding on a non-denaturing agarose gel. As shown in Figure 4e, a gradual shift of Malat1 FL RNAs was observed as the concentration of GSTSuv39h1 increases (from lanes 3 to 6); $1600 \mathrm{~nm}$ yielded the most evident shift (lane 6; see also Supplementary Figure S4b); GST protein alone (Figure 4e, lane 2) or a GST-MyoD protein (Supplementary Figure S4c) did not cause the shift. The above results confirmed the physical association between Malat1 FL and Suv39h1. In addition, consistent with the results from RNA pull-down assay in Figure 4d, GST-Suv39h1 protein was able to cause an evident shift of Malat1 F4 fragment and a relatively weaker shift of F2 and F3; such a shift appeared non-existent for F1 or negative control antisense Malatl (Figure 4e). When further mapping the interacting domain in Suv39h1 protein [44], we found that SET/Post-SET domain that catalyzes histone methyltransferase activity displayed evident binding with Malat1; Pre-SET domain or Chromodomain (chromatin-binding domain), whereas displayed no binding with Malat1 (Supplementary Figure S4d). Next, to answer the question whether Malat1 is tethered to the MyoD-binding DNA loci, Chromatin Isolation by RNA Purification (ChIRP) assay [45] with both odd and even tiling oligos against Malat1 was performed. We were able to specifically retrieve substantial amount of endogenous Malat1 transcripts from the promoter regions of MyoD/Suv39h1 target genes including Myogenin, Tnni2, MyHC and Ccnd3 (Figure $4 \mathrm{f}$ and Supplementary Figure S4e). Next, we performed ChIP-PCR assays to show that knockdown of Malat1 significantly impaired the binding of endogenous Suv39h1 protein to the MyoD-binding loci, including
Myogenin, MCK, Tnni2, MyHC, Ccnd3 and P21 promoter or enhancer (Figure 4g, Supplementary Figure S4f), confirming that Malat1 was critical for the recruitment of Suv39h1 to the MyoD target loci. In addition, the occupancy of HP1 $\beta$ and HDAC1 was also decreased on some of the above loci in the shMalat1 C2C12 cells (Figure 4h and i), even though Malat1 did not directly interact with them, suggesting that Malat1 may also affect the stability of the repressive complex. Suv39h1 is a methyltransferase that induces trimethylation of histone 3 lysine 9 (H3K9me3) at target sites. Expectedly, H3K9me3 levels at all the above target loci were significantly reduced upon Malat1 knockdown (Figure 4j). Interestingly, occupancy of MyoD on $M C K, M y H C$ and $C c n d 3$ was enhanced after knocking down Malat1 (Figure 4k), which was probably correlated with the induction of the genes. Although we could only detect increased Set7 occupancy on some sites (Supplementary Figure S4g), there was no significant increase in H3K4me1 despite the role of Set7 as a Histone 3 monomethylase [36], suggesting that the increased transcription following Suv39hl displacement may be mediated through other mechanisms. Altogether, the above results supported that Malat1 had a critical role in regulating MyoD trans-activation by recruiting Suv39h1 to the target loci. To further strengthen our findings, we showed that siMalat1 transfection into 10T1/2 cells reverted the suppressive effect of Suv39h1 on MyoD-induced target gene expression (Figure 41). The same rescuing effect can also be observed on MyoD-responsive myogenic reporters, Myog-Luc and 4RE-Luc (Figure 4m). These results further underscored the functional importance of Malat1 in modulating MyoD/Suv39h1 transcriptional activity.

\section{Malat1 depletion accelerates skeletal muscle differentiation in vivo}

Although no obvious developmental phenotype was observed in the recently generated Malat1-KO mice [38-40], we wanted to examine whether these mice would show any phenotype under certain circumstances, for example, the injury-induced muscle regeneration. In line with the previous report [40], the body size, weight and gross morphology of the KO mice displayed no obvious abnormality (Figure 5a and b). Further examination of their limb muscles did not reveal any evident difference from the WT littermates either, despite a sharp decrease in Malat 1 expression in $\mathrm{KO}$ muscles (Figure $5 \mathrm{c}$ and $\mathrm{d}$ ). However, in the subsequent analyses of the cardiotoxin-induced muscle regeneration process (Figure 5e), a subtle but notable 
a

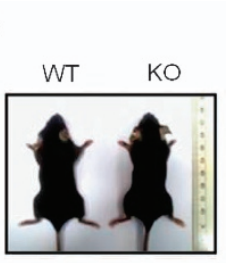

b

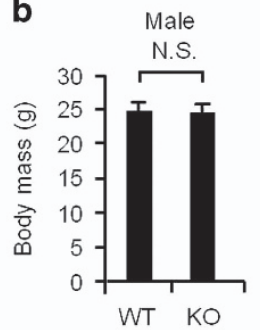

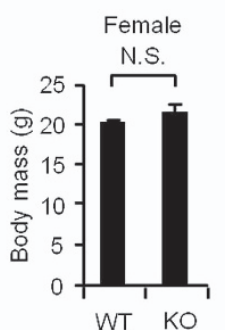

c

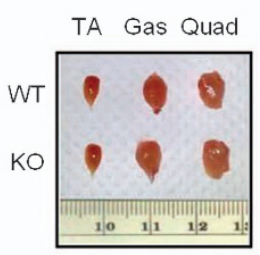

d

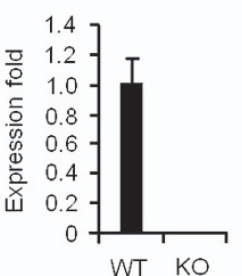

e
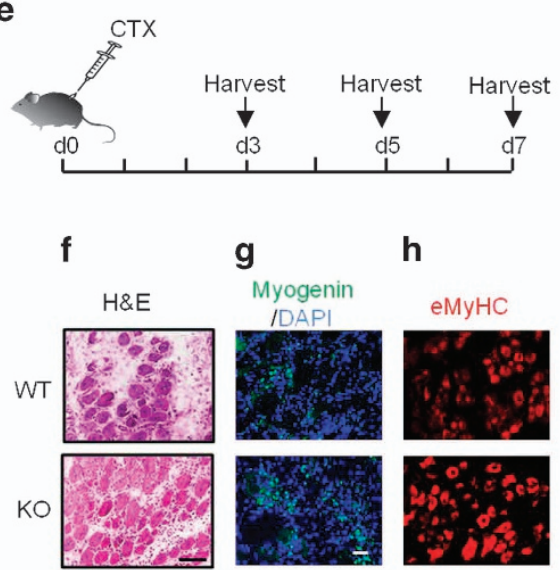

j

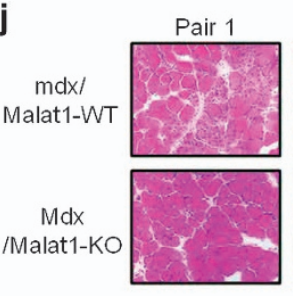

I Myogenin

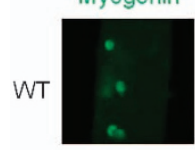

KO
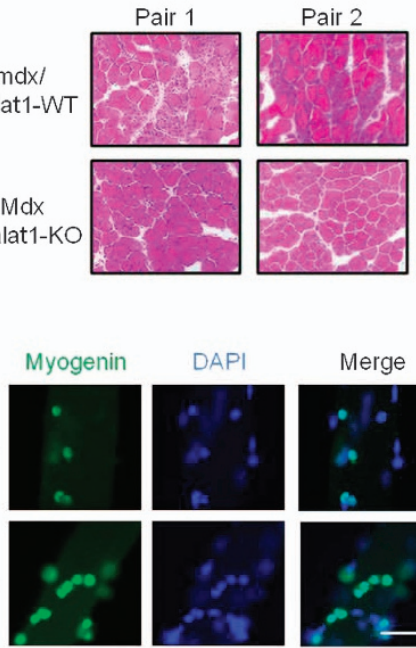

Pair 3

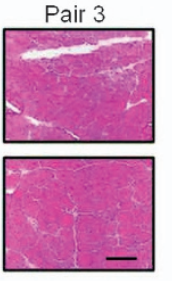

n Myogenin
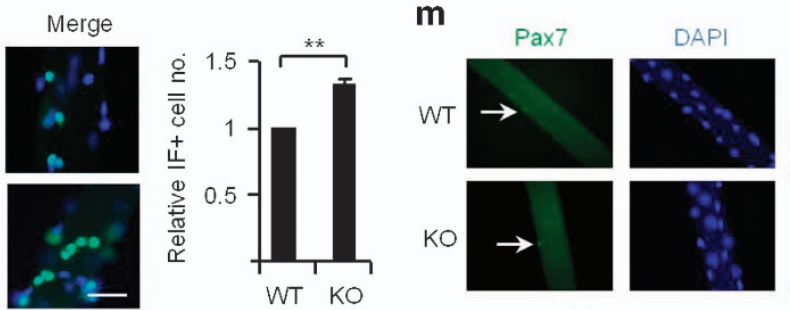

- Myogenin
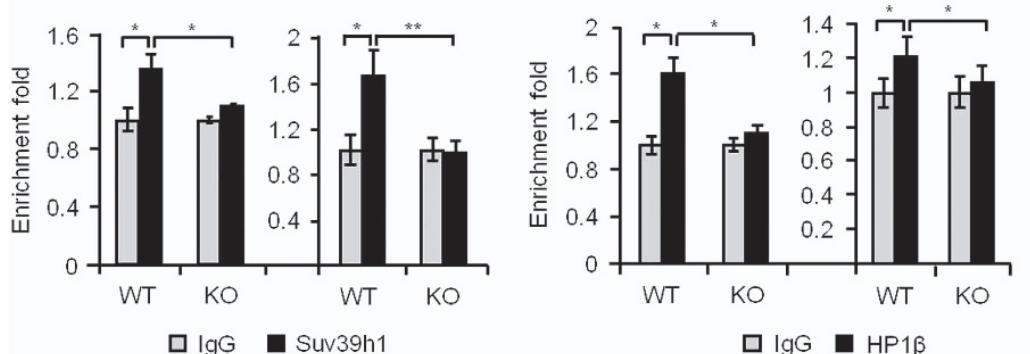

p Myogenin
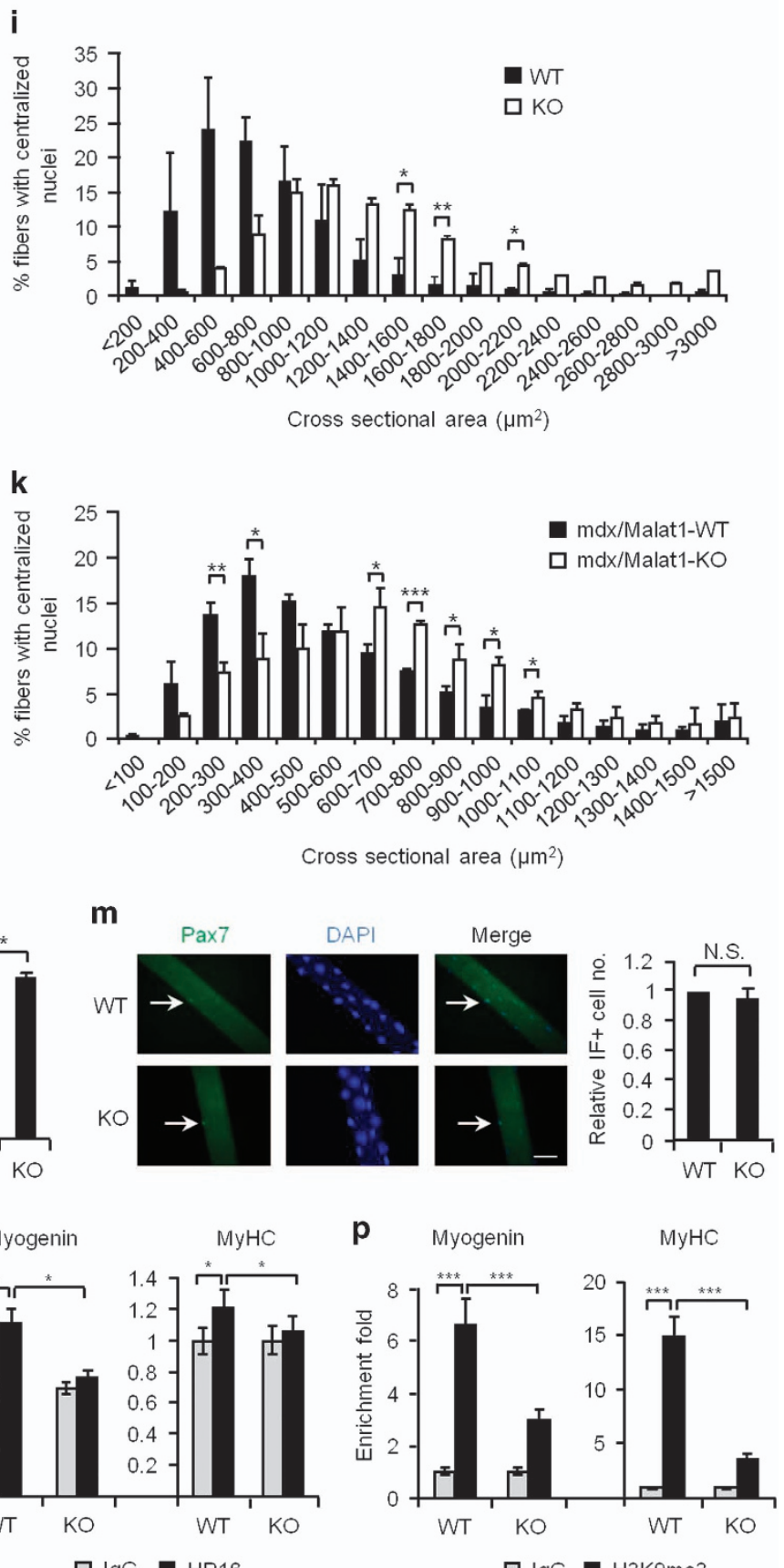

Cross sectional area $\left(\mu \mathrm{m}^{2}\right)$ 
acceleration was observed in the $\mathrm{KO}$ mice compared with WT littermates by hematoxylin and eosin (H\&E) staining of the muscle sections on day 7 post injury (Figure 5f). In the following immunostaining of the above sections, the increased number of cells positively stained for Myogenin and eMyHC also indicated enhanced muscle regeneration in Malat1-KO mice compared with WT littermates (Figure $5 \mathrm{~g}$ and $\mathrm{h}$ ). By measuring the cross-sectional areas (CSA) of the newly formed fibers with central nuclei, we found that the fiber size in $\mathrm{KO}$ mice was significantly larger compared with WT littermates (Figure 5i), confirming that the regeneration in $\mathrm{KO}$ mice was indeed accelerated. Interesting, heterozygous Malat1 mice did not display such accelerated muscle regeneration (Supplementary Figure S5a-d), indicating a lack of haploinsufficiency. To further validate our findings, we also examined the effect of Malat1 depletion in another regeneration model: the dystrophic muscle. We used the mdx mouse model of muscular dystrophy to generate $\mathrm{mdx} /$ Malat1-KO double-KO mice. Massive degenerationregeneration was observed in the $\mathrm{mdx}$ mice of 3-6 weeks of age (Figure 5j). As expected, enhanced muscle regeneration was observed in the $\mathrm{mdx} /$ Malat1KO mice compared with littermate mdx/Malat1-WT mice by H\&E staining on cross-sections of TA muscle from 5-week-old mice (Figure 5j). By calculating the CSA of fibers with centrally localized nuclei we found an increased number of fibers with larger CSA in $\mathrm{mdx} /$ Malat1-KO mice than that in $\mathrm{mdx} /$ Malat1-WT mice
(Figure 5k). To further test whether the above phenotype stemmed from the enhanced SC activities, we isolated single muscle fibers with SCs attached as an ex vivo model [30]. When induced to differentiate in the culture, SCs from the KO fibers displayed a higher myogenic competence as shown by increased Myogenin staining compared with the WT (Figure 51, 1.3 -fold increase of the positively stained cells). This is consistent with what we observed in $\mathrm{C} 2 \mathrm{C} 12$ cells with Malat1 knockdown (Figure 2), suggesting the increased myogenic capacity of the SCs contributed to the enhanced muscle regeneration in the $\mathrm{KO}$ mice. Interestingly, by staining for $\operatorname{Pax} 7$ on the freshly isolated fibers or on the cross-sections of TA muscles, we did not detect significant difference in the number of SCs in KO versus WT mice (Figure $5 \mathrm{~m}$ and Supplementary Figure S5e), suggesting that Malat1 depletion did not affect the number of quiescent SC pool. To further test whether the molecular mechanism uncovered from $\mathrm{C} 2 \mathrm{C} 12$ myoblast applies in vivo in the $\mathrm{SC}$ cells, we performed in vivo ChIP for Suv39h1 or HP1 $\beta$ in the cardiotoxin-induced regenerating muscle from WT or KO mice. Reduced occupancy of Suv39h1 and HP1 $\beta$ at Myogenin and $M y H C$ promoters was observed (Figure $5 \mathrm{n}$ and o); consistently, decreased level of $\mathrm{H} 3 \mathrm{~K} 9 \mathrm{me} 3$ at these sites was also detected (Figure 5p and Supplementary Figure S6). Taken together, our data revealed that Malatl depletion in mice led to accelerated muscle regeneration through boosting the myogenic potential of SCs.

Figure 5 Malat1 depletion leads to enhanced regeneration in acute or dystrophic injured mouse muscles. (a) Comparison of WT and Malat1-KO adult littermates showed no apparent difference. Representative images of one pair of mice were shown. (b) No difference was detected comparing the body mass of WT and KO male/female littermates or (c) comparing the size of three types of mouse limb muscles (Tibialis anterior, TA; Gastrocnemius, Gas; Quadriceps, Quad). (d) QRT-PCR detection of the Malat1 expression in the TA muscles from WT or KO mice. (e) The scheme for CTX injection into Malat1-WT or KO mouse TA muscle and the tissue harvesting time points for subsequent analyses. (f) The above injected muscles were harvested on day 5 post injections and used for H\&E staining. The images were taken at $\times 200$ magnification. Scale bar $=100 \mu \mathrm{m} .(\mathbf{g}, \mathbf{h})$ The above CTX-injected muscles were harvested on day 3 post injections, which were used for immunostaining of Myogenin or eMyHC, respectively. The images for Myogenin were taken at $\times 100$ magnification. The images for eMyHC were taken at $\times 200$ magnification. Scale bar $=100 \mu \mathrm{m}$. (i) The cross-sectional area of the newly formed fibers on day 7 post-CTX injection with centralized nuclei was quantitated on the H\&E staining sections using ImageJ (NIH, Bethesda, MD, USA). Three pairs of WT and KO mice were analyzed. Data are presented as mean $\pm \mathrm{s}$.d. The $P$-value was calculated with Student's $t$-test. (j) The TA muscles of mdx/Malat1-KO mice and littermate mdx/Malat1-WT mice were harvested at 5 weeks postnatal and used for H\&E staining. Images from three pairs of representative mice are shown. Scale bar $=100 \mu \mathrm{m}$. (k) The crosssectional area of fibers with centralized nuclei was measured from the above H\&E images. Three pairs of littermate mdx/Malat1-WT and $\mathrm{mdx} /$ Malat1-KO mice were analyzed. Data are presented as mean \pm s.d. The $P$-value was calculated with Student's $t$-test. (I) Single myofibers were isolated from the extensor digitorum longus muscle of 2-month-old littermate WT and KO mice and cultured for $72 \mathrm{~h}$ before staining for Myogenin (green) and DAPI (blue). The number of Myogenin+ SCs was counted from three pair of littermates. Scale bar $=50 \mu \mathrm{m}$. $(\mathbf{m})$ The above myofibers were fixed immediately after the isolation and used for IF staining of Pax7 (green) and DAPI (blue). The number of $\mathrm{Pax}^{+}$satellite cells was counted from three littermates. Scale bar $=100 \mu \mathrm{m}$. (n-p) ChIP-PCR analyses of Suv39h1, HP1 $\beta$ and H3K9me3 enrichment on the Myogenin or MyHC promoter in vivo using WT or KO TA muscle harvested 3 days postCTX injection. The IP efficiency was calculated by setting IgG value as one. All PCR data were normalized to GAPDH mRNA and represent the average of three independent experiments \pm s.d. ${ }^{*} P<0.05,{ }^{* *} P<0.01,{ }^{* * *} P<0.001$. 
Nuclear Malat1 is directly targeted by miR-181alAgo2 machinery upon myoblast differentiation

The interesting downregulation of Malatl at the very early stage of myogenic differentiation suggested that its expression must be tightly regulated. miRNA-lncRNA interaction is emerging as an important phenomenon. Although many studies favor the competing endogenous model, that is lncRNA acting as the sponges for miRNA to regulate its function, we thought about the alternative, that is, miRNA targeting Malat1 for its downregulation because exploration of the Ago2 CLiP-seq (photo-crosslinking immunoprecipitation using Ago2 antibodies, followed by deep-sequencing of RNAs) in mouse embryonic stem cells [46] revealed a potential association of Ago2 protein with Malat1 transcript (data not shown), suggesting that it could be bound and regulated by the miRNA/Ago2 silencing machinery (Figure 6a). We thus looked for miRNAs that could potentially target Malat1 upon differentiation; this led to the discovery of a sequence complementarity between Malat1 and 82 miRNAs (Supplementary Table S2) including miR-181a (Figure 6a), which happens to be a wellknown regulator of skeletal myogenesis [47]. Indeed, by cloning the identified miR-18a binding region in Malat1 into a luciferase reporter vector, we found that transfection of precursor oligos of miR-181a (pre-181a) but not a negative control or an irrelevant miR-193a downregulated the reporter activity (Figure 6b); moreover, mutating the predicted binding site (Figure 6a, Mutant) abolished this targeting effect (Figure 6b), suggesting that indeed miR-181a directly targeted Malat1 through the above-identified site. In line with the finding, overexpression of miR-181a by transfecting the precursor miR-181a oligos led to a significant downregulation of the endogenous Malat1 in differentiating $\mathrm{C} 2 \mathrm{C} 12$ cells (Figure $6 \mathrm{c}, 60 \%$ decrease, Supplementary Figure S7a and b), whereas depletion of miR-181a by Antagomirs (Anti-181a) increased the expression of Malat1 (Figure 6d, 1.5-fold, Supplementary Figure S7c). In contrast to the notable downregulation of Malat1 expression in the early stage of C2C12 differentiation (Figure 1d), miR-181a expression level was found to gradually increase (Figure 6e), supporting their functional antagonization during this stage. Indeed, as previously reported [47], miR-181a is known for its pro-myogenic function in myoblast cells. Still, we were uncertain about whether this miR-181a/Malat1 regulation could occur in the nucleus where Malat1 was enriched, given that the miRNA-mediated silencing machinery is traditionally thought to mainly function in the cytoplasm. To eliminate our doubts, we examined the subcellular localization of miR-181a and detected a substantial amount of miR-181a in the nuclear portion (Figure 6f). Consistently, we also detected a high amount of Ago2 protein in $\mathrm{C} 2 \mathrm{C} 12$ nuclei (Figure $6 \mathrm{~g}$ ), indicating that the miRNA/Ago 2 machinery could possibly function in the nucleus of myoblast cells. To further demonstrate the involvement of Ago2 in this regulation, we showed that biotinylated FL Malat1 transcripts could pull down an evident amount of Ago2 protein from nuclear lysates of early-differentiating $\mathrm{C} 2 \mathrm{C} 12$ myoblasts (Figure 6h); moreover, antibodies against Ago2 also retrieved a significant level of endogenous Malat1 transcripts from nuclear lysates ( Yaml and 7SK were not retrieved; Figure 6i), indicating an association between Ago2 protein and Malat1 RNAs in the C2C12 nucleus upon differentiation. In keeping with this, knockdown of Ago2 markedly increased the expression of Malat1 in differentiating myoblasts (Figure 6j). To further explore how miR-181/Ago2 downregulates Malat1 transcripts, we first tested whether they could suppress Malatl transcription as increasing lines of evidence showed the non-canonical functions of nuclear miRNA/Ago in recruiting epigenetic silencing complex, which triggered transcriptional silencing [48]. However, by nuclear run-on assay, we did not detect a decrease in nascent Malat1 transcription by miR-181a overexpression, suggesting that the regulation may not be at the transcriptional level; instead, miR-181/Ago2 may cause Malatl degradation through an nRISC machinery (Figure 6k). To test this notion, we found that, besides Ago2, multiple components of RISC including Dicer, Trbp, which were required for the recruitment of Ago2 to Dicer [49], and Tnrc6a/ GW182, which navigated Ago2 to translocate into the nucleus [50], were detected in the nuclear portion of C2C12 cells (Figure 61). Using RIP (Figure 6m) and RNA pull down (Supplementary Figure S7d) assays an association of these proteins with Malat1 transcripts was also detected in the $\mathrm{C} 2 \mathrm{C} 12$ nuclear lysates. The above findings supported the presence of nRISC in the $\mathrm{C} 2 \mathrm{C} 12$ nucleus and its participation in degrading Malat1 transcripts upon myoblast differentiation. Lastly, to confirm the functionality of the miR-181a/ Malat1 regulation, we further demonstrated that knockdown of Malat1 could revert the anti-myogenic effect of anti-miR-181a treatment on myogenesis (Figure 6n). Altogether, the above data suggested that Malat1 was reduced by miR-181a/Ago2 degradation through the nRISC-dependent machinery in the nucleus of myoblast cells upon early differentiation. 
a

miR-181a: 3' ugagUGGCUGUC---GCAACUUACAa 5,

Malat1 : 3916 5' aaagACCUGUAGAGCUGUUGGAUGUU 3, 3941 (WT)

Malat1

(Mutant)

5' aaagACCUGUAGAGCUGCCUGCGACU 3,
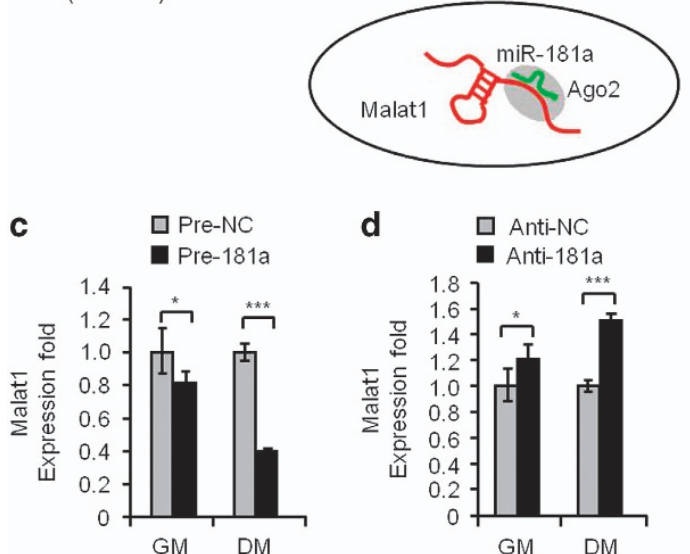

e
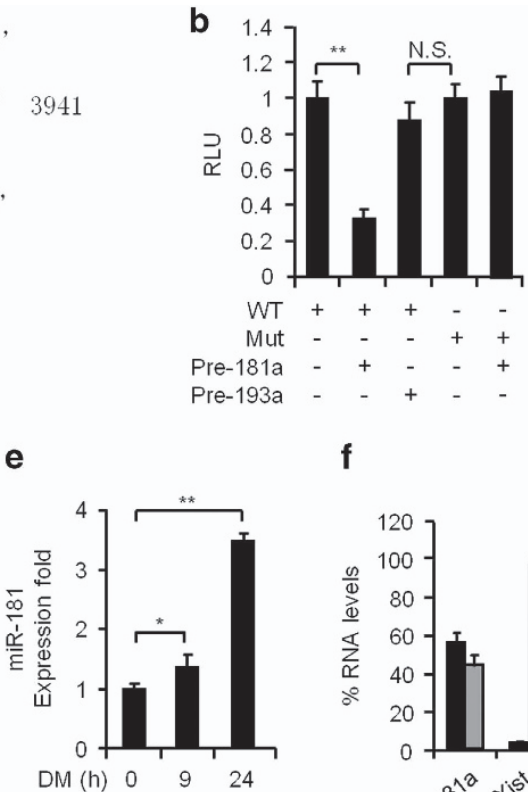

f

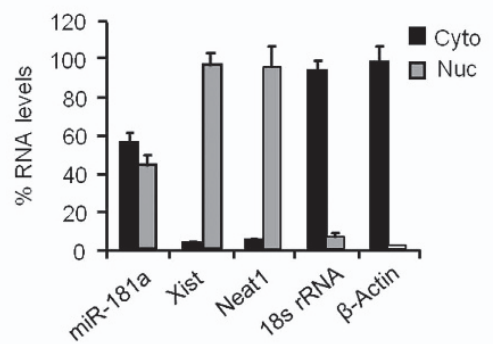

g

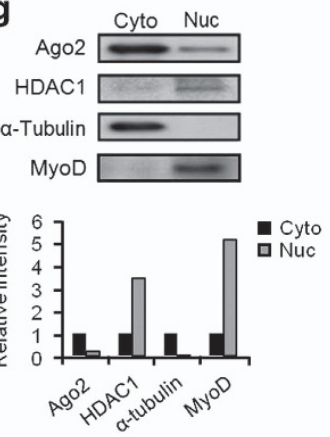

h

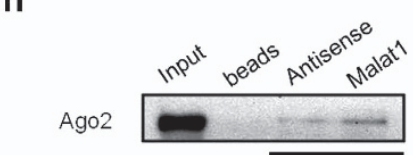

in vitro transcribed RNA

i

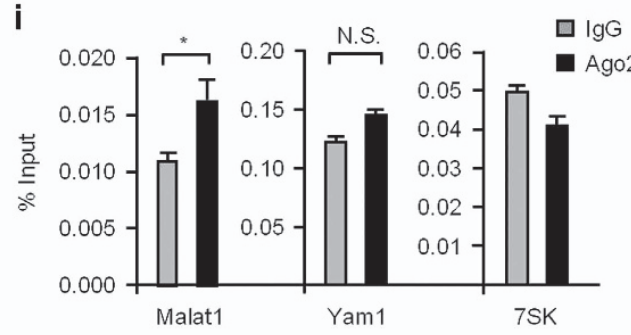

$\mathbf{k}$

$\rightarrow+460+1236 \quad+4545+5875+6576$
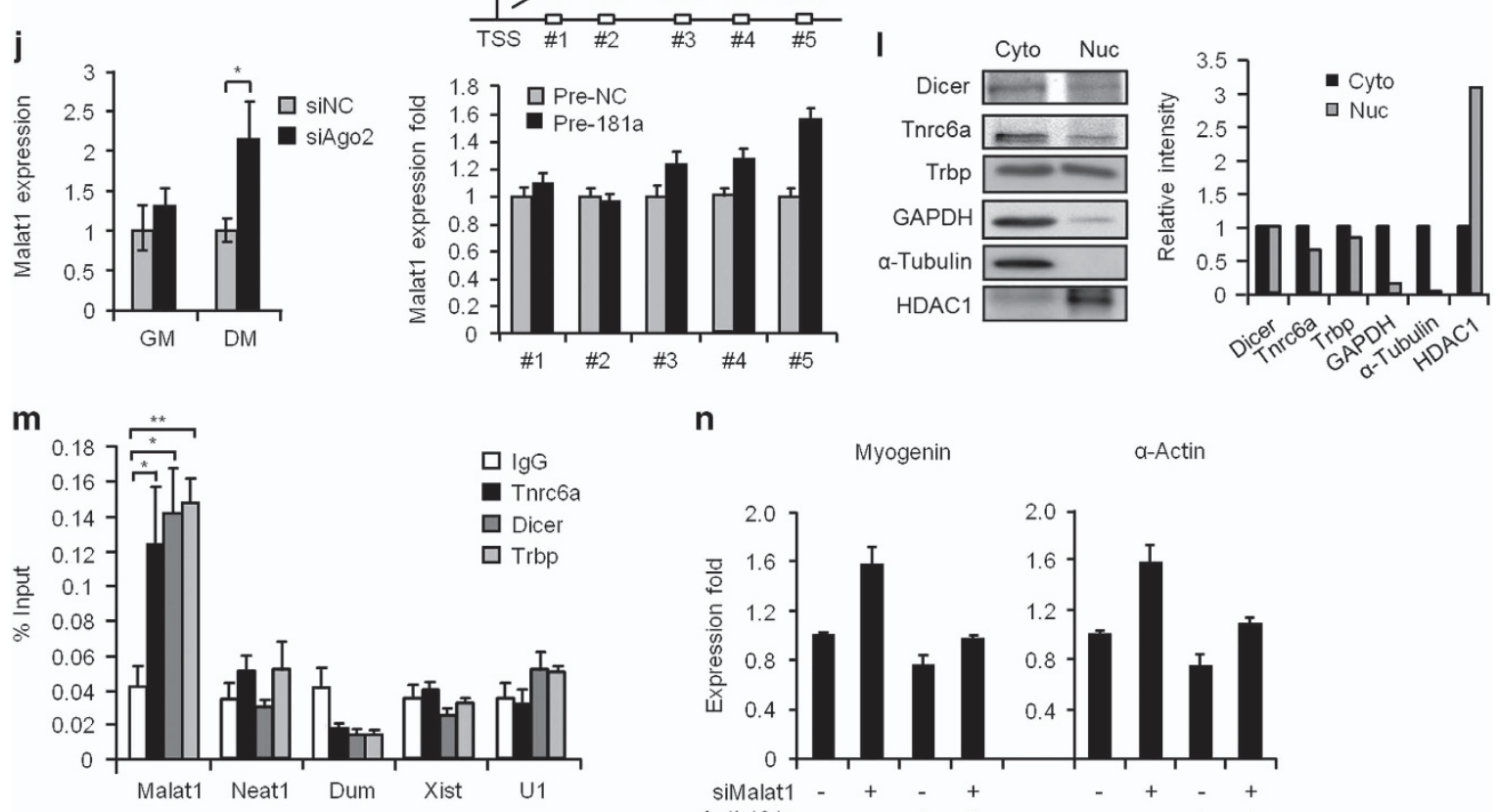

n

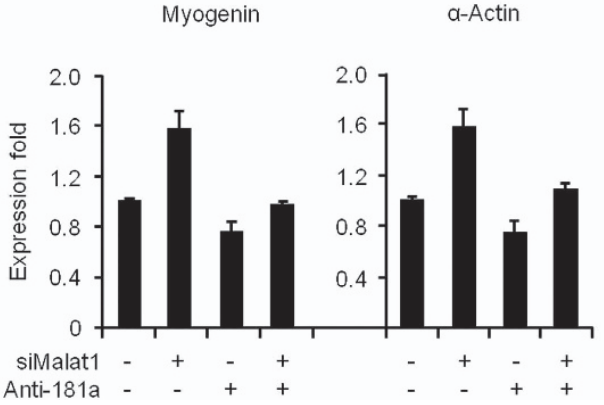




\section{Discussion}

Our findings provided a comprehensive view of Malat1 function in skeletal muscle cells and muscle regeneration, and also elucidated a novel role of Malat1 in the epigenetic/transcriptional regulation of gene expression. As illustrated in Figure 7, upon injury, SCs are activated into committed myoblasts that proliferate and further differentiate into myotubes. In the proliferating myoblasts Malat1 is present in high abundance and recruits Suv39h1 to MyoD-binding loci through its ability to tether with the MyoD target loci; subsequent formation and stabilization of the Suv39h1/HDAC1/HP1 $\beta$ complex leads to trimethylation of Histone 3 lysine 9 (H3K9me3) and repression of the target gene expression. At the onset of differentiation, miR-181a expression is induced, causing Malat1 degradation through their direct interaction and Ago2-dependent nRISC machinery in the nucleus. The repressive complex is thus destabilized and replaced by Set7-containing activating complex, which allows MyoD trans-activation to occur on the target loci. The myogenic differentiation represents a critical step during the entire muscle regeneration, which is attributed to SCs.

Malat1 is no doubt one of the first identified and one of the most well-studied lncRNAs. Despite numerous reports on its abundance in various cell types and overexpression in many human tumors, the exact cellular function of Malat1 is still a matter under intense investigation. It is most popularly known for its regulation of RNA splicing through interacting with SR proteins, and is less known as an epigenetic modulator. However, Yang et al. [13] showed Malat1 can bind to histone methyltransferases/demethylases such as SET2, although no further functional characterization was carried out. Our study is the first to show that Malat1 interacts with Suv39h1, which is also a Set domain-containing methyltransferase, and provides detailed functional characterization of this interaction. Distinct from the gene-activating role reported in the above study, Malat1 association with Suv39h1 leads to gene repression, underscoring its pleiotropic functions in gene regulation.

Our findings from using various protein/ RNA-binding assays demonstrate a direct physical interaction between Malat1 FL transcript and Suv39h1 protein (Figure 4). Knockdown of Malat1 also hampered the association of the entire MyoD/Suv39h1/ HP1 $\beta / H D C A 1$ complex with the target loci, suggesting that Malat1 probably is important for the recruitment of Suv39h1 and stabilization of the complex on the target loci. In addition, by ChIRP assay (Figure 4f), we showed that Malat1 is physically tethered to the

Figure 6 Malat1 is directly targeted by miR-181a in the nucleus of myoblast through a Ago2-dependent nRISC machinery. (a) Upper: the predicted binding site between miR-181a and Malat1-WT sequence (5'-3916-3941-3'). A mutant was generated by mutating the seed sequence (UUGAAUGU, underlined) to CCUGCGAC. Lower: the hypothetical model depicting that Malat1 transcript is directly targeted by miR-181a/Ago2 machinery in the myoblast nucleus. (b) The above-identified WT or mutated miR-181a-binding site was cloned into a pMIR luciferase reporter vector and transfected into the C2C12 cells with miR-181a (pre-181a) or miR-193a (pre-193a) precursor oligos. Luciferase activities were determined $48 \mathrm{~h}$ post transfection. (c) Pre-181a or negative control (Pre-NC) precursor oligos were transfected into $\mathrm{C} 2 \mathrm{C} 12$ cells. The expression level of Malat1 was examined by qRT-PCR after the cells were switched to DM or maintained in the growth medium (GM) for $48 \mathrm{~h}$. (d) An antagomiR of miR-181a (Anti-181a) or a negative control (Anti-NC) oligo was transfected into $\mathrm{C} 2 \mathrm{C} 12$ and the expression of Malat1 was determined as above. (e) The expression level of miR-181a during C2C12 differentiation at 0 , 9 and $24 \mathrm{~h}$ was determined by qRT-PCR. (f) The distribution of miR-181a in C2C12 cytoplasmic (Cyto) or nuclear (Nuc) fraction was determined by qRT-PCR. Xist, Gapdh and U6 RNAs were used as controls to compare with. (g) Upper panel: cytoplasmic or nuclear level of Ago2 proteins was detected by western blotting. MyoD and HDAC1 were used as nuclear protein controls and $\alpha$-Tubulin was used as cytoplasmic protein control. Lower panel: relative quantification of the band intensity was plotted. (h) RNA pull-down assay was performed with biotinylated Malat1 FL transcripts to retrieve Ago2 in C2C12 nuclear lysates. Beads only or FL antisense transcripts were used as negative controls. The in vitro generated transcripts were visualized on the Agarose gel to show equal loading. (i) The $\mathrm{C} 2 \mathrm{C} 12$ nuclear lysate was immunoprecipitated by an Ago2 antibody. The retrieved Malat1 RNAs were detected by qRT-PCR. Yam1 and 7SK RNAs were also detected as controls. (j) Ago2 was knocked down by an siRNA oligo in C2C12 myoblast and the Malat1 expression was examined by qRT-PCR after the cells were switched to DM or maintained in GM for $48 \mathrm{~h}$. (k) Nuclear run-on assay was performed in C2C12 nuclei transfected with pre-181a or pre-NC oligos. Primers were designed to detect nascent transcription from five different locations of the Malat1 gene. (I) Left: cytoplasmic or nuclear levels of Dicer, Tnrc6a and Trbp proteins were detected by western blotting. GAPDH and $\alpha$-Tubulin were used as cytoplasmic protein control and HDAC1 was used as nuclear protein control. Right: relative quantification of the band intensity was also plotted. $(\mathbf{m})$ The nuclear extract of $\mathrm{C} 2 \mathrm{C} 12$ cells was immunoprecipitated by the indicated antibodies. The retrieved Neat1, Dum, Xist, U1 or Malat1 RNAs were detected by qRT-PCR. (n) C2C12 cells were transfected with the indicated oligos. Expression of the indicated myogenic genes was analyzed by qRT-PCR. All PCR data were normalized to GAPDH mRNA and represent the average of three independent experiments \pm s.d. All luciferase data were normalized to Renilla luciferase activities and represent the average of three independent experiments \pm s.d. ${ }^{*} P<0.05,{ }^{* *} P<0.01,{ }^{* * *} P<0.001$. 
recruitment site, that is, the promoter or enhancer region of the myogenic genes but exactly how the RNA/DNA interaction occurs needs further investigation. It is likely that the binding may depend on contact with some component of the transcription process and is not because of DNA sequence alone as CHART analysis has identified MALATl enriching at active genes [51]. In addition, we also mapped the functional binding domain of Malat1 with Suv39h1 through deletion mapping. Interestingly, results from both RNA pull-down and RNA/protein EMSA assays indicated that the $3^{\prime}$ domain (5 185-6982) seems to be sufficient for efficient Suv39h1 binding. The $3^{\prime}$ end of Malat1 is not produced by canonical cleavage/polyadenylation but instead by recognition and cleavage of a tRNA-like structure by RNAse P [52]. The processed Malat1 transcript is protected from $3^{\prime}$ to $5^{\prime}$ exonucleases by highly conserved triple helical structures at the $3^{\prime}$ end, which promotes its RNA stability and high expression in most cell types [52]. Xu et al. [53] also identified the $3^{\prime}$ end of Malat 1 as an important motif in the invasion and metastasis of colorectal cancer. Nevertheless, the other domains (F1, F2 and F3) could also interact with Suv39h1 to various degrees, which is not surprising considering each fragment used in the mapping is large enough to form complex secondary structures. Interestingly, we also mapped the SET domains but not the chromodomain of Suv39h1 as the interacting domain with Malat1. This is consistent with the previous reports [54, 55] showing SET-containing proteins of the SET1 and SET2 families contain motifs in the pre-SET region or at the pre-SET/SET and SET/post-SET boundaries, which very tightly bind single-stranded DNA and RNA. Yang et al. [13], however, think it is the chromodomain of the Pc2 protein that binds with Malat1 to function as modulators of Pc2 for 'reading' the histone code in HeLa cells. Further structural analysis will be needed to better understand the structure-function relationship.

Using myoblast differentiation as a model, our study mainly elucidated an inhibitory role of Malat 1 in the myogenic differentiation. However, Malat1 may exert pluripotent functions in other aspects of SC activities. Of note, we observed a delay in myoblast proliferation upon Malat1 knockdown, suggesting that it may also have a pro-proliferative function in myoblasts, which is in keeping with reports linking Malat1 to cell cycle progression [56]. Moreover, it will be interesting to further explore the cellular mechanisms underlying these other functions in SCs. In addition, although

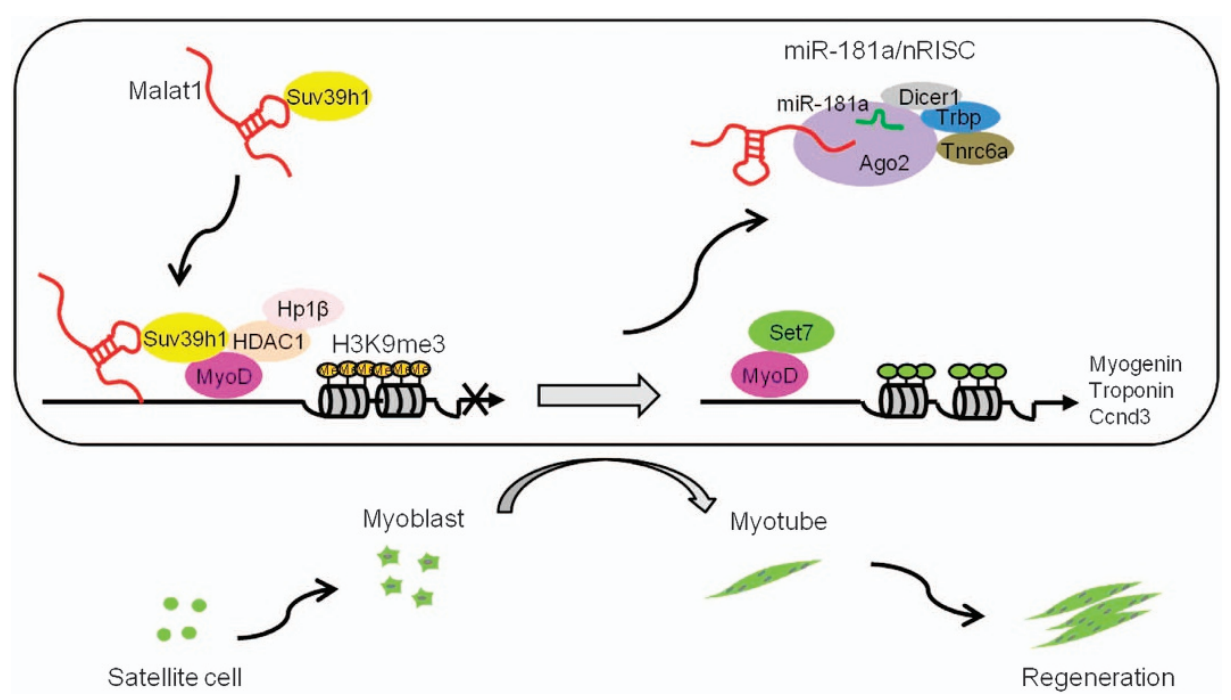

Figure 7 Schematic model of Malat1 regulation of MyoD transcriptional activity during myogenesis. The model depicts the role of the miR-181a-Malat1-MyoD/Suv39h1 regulatory axis in myogenic differentiation and muscle regeneration. Upon injury, SCs are activated into committed myoblasts, which proliferate and differentiate into myotubes, leading to successful regeneration. In the proliferating myoblasts Malat1 is present in high abundance and recruits Suv39h1 to MyoD-binding loci through tethering to the DNA loci; the subsequent formation and stabilization of the Suv39h1/HDAC1/HP1 $\beta$ complex leads to trimethylation of the Histone 3 lysine 9 (H3K9me3) and repression of the target gene expression. Upon differentiation, miR-181a expression is induced, causing Malat1 degradation through their direct interaction and Ago2/nRISC-dependent machinery. The repressive complex is thus destabilized and replaced by Set7-containing activating complex, leading to monomethylation of Histone 3 lysine 4 (H3K4me1) and target gene activation. The myogenic differentiation represents a critical step during the entire muscle regeneration. 
Malat1 expression is quickly downregulated at the early stage of differentiation, it sharply recovers when the myoblasts enter terminal differentiation (Figure 1d), suggesting that Malat1 may have additional functions in promoting myotube fusion or maintaining myotube homeostasis. We must point out that in contradictory to our findings, Watts et al. [31] showed a slight decrease in Myogenin RNA after Malat1 knockdown, leading to the conclusion that Malat1 is a pro-myogenic factor. In a separate study, Han et al. [32] also reported reduced Myogenin mRNA expression in differentiating myotubes in response to partial Malat1 inhibition. In both studies, the measurement was conducted 4 days into the differentiation whereas Myogenin induction usually occurs in the early phase. In addition, because of its anti-proliferative effect in the myoblast cells, it is necessary to adjust the cell confluence to comparable levels when inducing cell differentiation for the in vitro experiments, which could be another confounding factor for causing the discrepancy seen in our studies. Nevertheless, in addition to the multiple lines of evidence from using in vitro cell culture, we have also used knock-out mice model to strengthen our findings showing the anti-differentiation role of Malat1, which is consistent with a recent report that inhibition of Malat1 could induce cancer cell differentiation [57]. We thus believe that our study provide substantial evidence to support the anti-myogenic action of Malat1 during the early phase of differentiation. Still, Malat1 is one of the multifunctional lncRNAs in skeletal muscle cells, and continuous exploration will bring many future surprises.

Given its known important functions in many cells, the high abundance in most cell types examined so far and its strong sequence conservation from human to zebrafish, it is surprising that the Malat1-KO mouse did not exhibit any obvious developmental phenotype. Our study is the first to report that Malat1-KO mouse indeed displays some phenotype in skeletal muscle tissues under stress (acute or chronic injury-induced regeneration), even though the skeletal muscle development appears normal. Although not dramatic, the accelerated regeneration was repeatedly seen in the KO mice, confirming that Malat1 acted to repress the regeneration process. This can be attributed to some extent to its regulation of the SC myogenic ability as the SCs isolated from the $\mathrm{KO}$ mice displayed accelerated myogenic differentiation. However, we cannot rule out the possibility of reduced degeneration as well as contributions from other cell types in the injured muscle as regeneration is a process occurring in a complex niche environment and governed by intercalating signaling networks. In order to better elucidate the functional impact of its loss on SCs, it will be necessary to generate an SC-specific KO mouse model of Malat1 in the future. It is also foreseeable that Malat1-KO mice may have other tissue- or condition-specific phenotypes that await to be discovered. Indeed, two recent reports published during the preparation of our manuscript demonstrated that Malat1-KO mice showed a delayed vessel extension in the retina [58] and decreased fertility [59] compared with WT littermates.

Although the high abundance and stability of Malat1 could suggest a competing endogenous function titrating the amount of miR-181a, emerging reports start to demonstrate that the microRNAs binding could also reduce lncRNA stability [14]. Nonetheless, there is still limited mechanistic understanding for how miRNA-mediated targeting and degradation of lncRNAs occur mainly because canonically miRNA is believed to regulate mRNA expression through RISC machinery in the cytoplasm. However, in recent years several lines of evidence point to additional functions for miRNAs in the nucleus that have been largely ignored so far [48]. For example, the majority of cellular miRNAs are present in both the nucleus and the cytoplasm. Multiple components of the RISC machinery are present in the nuclear compartment. Our results clearly demonstrate that miRNAs can indeed target nucleus-residing lncRNAs for degradation through the nRISC machinery and highlights that the complexity of the non-coding RNA involved regulatory interactions.

Modulating the activities of transcription factors and epigenetic regulators on myogenic loci is crucial for skeletal muscle differentiation. Our findings underscore the important role of Malat1 in this process. It is one of the few lncRNAs identified so far that have important functions in myogenesis; we nevertheless believe that many such lncRNAs exist and will need to be characterized in the near future. Together with transcription factors and chromatin regulators, they form complex regulatory networks orchestrating the precise gene transcription. Better knowledge of their acting mechanisms will enable the understanding and modulation of muscle regeneration in pathophysiological conditions.

\section{Materials and methods}

\section{Cell culture}

Mouse C2C12 myoblast cells (CRL-1772) and C3H/10T1/2 (10T1/2) fibroblast cells (CCL-226) were obtained from ATCC 
and cultured in DMEM medium (12800-017, Gibco) with $10 \%$ fetal bovine serum (10270-106, Gibco), 100 units $/ \mathrm{ml}$ of penicillin and $100 \mu \mathrm{g}$ of streptomycin (15140-122, Gibco) (growth medium, or GM) at $37^{\circ} \mathrm{C}$ in $5 \% \mathrm{CO} 2$. To induce cell differentiation, near confluent cells were switched to DMEM containing $2 \%$ horse serum (16050-122, Thermo Fisher Scientific) (differentiation medium, or DM). Muscle satellite cells (SCs) of 2 months old Pax7-nGFP mouse [60] were isolated as previously reported [61]. The $\operatorname{Pax} 7$ positive cells were sorted based on the GFP signals. Sorted-out SCs were collected upon sorting as quiescent SCs or cultured in F10 medium (F0723, Merck Millipore) with 10\% horse serum (26050088, Invitrogen, Carlsbad, CA, USA).

\section{Cell proliferation assay}

MTT assays were conducted following the instructions of manufacturer (M6494, Invitrogen). Culture volume (1/10) of MTT solution $\left(5 \mathrm{mg} \mathrm{ml}^{-1}\right.$ ) was added to cultured cells incubated in a $\mathrm{CO}_{2}$ incubator at $37^{\circ} \mathrm{C}$ for $4 \mathrm{~h}$. After the incubation medium is removed and DMSO was added to dissolve the crystals with pipetting up and down. A volume of $100 \mu 1$ of DMSO solution was transferred to a 96-well plate, and the absorbance was measured at $550 \mathrm{~nm}$ with background subtraction at $650 \mathrm{~nm}$. BrdU assay was conducted as the manufacturer's instructions (B5002, Sigma-Aldrich). Growing cells on coverslips were incubated in culture medium with $10 \mu \mathrm{M}$ BrdU for $20 \mathrm{~min}$. Cells were then washed for three times in phosphate-buffered saline (PBS) and fixed with fixative solutions (3 volumes of glycine solution (50 mM, $\mathrm{pH} 2.0$ ) with 7 volumes of pure ethanol) for $45 \mathrm{~min}$ at room temperature (RT). Cells were washed in PBS twice and then incubated in $4 \mathrm{M} \mathrm{HCl}$ for 10-20 min at RT to denature DNA. Cells were washed for three times to neutralize the $\mathrm{pH}$ and then incubated in the incubation buffer (PBS containing $0.5 \%$ bovine serum albumin and $0.1 \%$ Tween 20) to block unspecific binding. Anti-BrdU antibody was then added into the incubation buffer; after $1 \mathrm{~h}$ incubation at RT, cells were washed in PBS. The coverslips were then mounted with antifade mounting solution with 4,6-diamidino-2phenylindole (DAPI; P36931, Invitrogen). Pictures were captured with a fluorescence microscope (Zeiss, Jena, Germany).

\section{Cell fractination}

RNA from different subcellular fractions of $\mathrm{C} 2 \mathrm{C} 12$ cells were isolated as previously described [62]. Cell pellet was lysed with $175 \mu \mathrm{l}$ per $10^{6}$ cells of cold RLN1 solution $(50 \mathrm{~mm}$ Tris $\mathrm{HCl} \mathrm{pH}$ $8.0 ; 140 \mathrm{~mm} \mathrm{NaCl} ; 1.5 \mathrm{~mm} \mathrm{MgCl}_{2} ; 0.5 \% \mathrm{NP}-40 ; 2$ mм Vanadyl Ribonucleoside Complex) and incubated $5 \mathrm{~min}$ on ice followed by centrifuge at $300 \mathrm{~g}$ and $4{ }^{\circ} \mathrm{C}$ for $2 \mathrm{~min}$. The supernatant was saved as cytoplasmic fraction. The pellet was resuspended in $175 \mu \mathrm{l} / 10^{6}$ cells of cold RLN2 solution (50 mM Tris $\mathrm{HCl} \mathrm{pH}$ 8.0; $500 \mathrm{~mm} \mathrm{NaCl} ; 1.5 \mathrm{~mm} \mathrm{MgCl} 2 ; 0.5 \%$ NP-40; 2 mм Vanadyl Ribonucleoside Complex) and incubated on ice for $5 \mathrm{~min}$. The suspension was then centrifuged at $4{ }^{\circ} \mathrm{C}$ and $16360 \mathrm{~g}$ for $2 \mathrm{~min}$ and the supernatant was saved as nuclear-soluble fraction and the pellet was kept as the nuclear-insoluble fraction. RNAs from different fractions were isolated by Trizol (15596018, Invitrogen).

\section{Single myofiber isolation}

Single myofibers were isolated as previous described [61]. Briefly, the extensor digitorum longus muscles were carefully cut from tendon to tendon and digested in F10 medium containing Collagenase type II $\left(800 \mathrm{U} \mathrm{ml}^{-1}\right)$ at $37^{\circ} \mathrm{C}$ in a water bath for $75 \mathrm{~min}$. After digestion, large bore pasteur pipette was used to flush the muscles in warm medium to release myofibers. Live single myofibers were transferred to a new dish and the operation was repeated for two to three times for removing dead fibers and debris. Isolated single myofibers were further cultured in F10 medium with $10 \%$ horse serum.

\section{Plasmids}

The mouse Malat1 FL expression plasmid was obtained from Dr Kannanganattu V Prasanth [11]. The mouse Malat1 FL plasmid used for in vitro transcription was obtained from Dr Shinichi Nakagawa. Gal4-MyoD and Gal4 luciferase reporter plasmids were obtained from Dr Zhenguo Wu. The Suv39h1 expression plasmid was obtained from Dr Asoke K Mal [35]. The plasmids expressing full length or fragments of Suv39h1-GST, or GST only in bacteria were obtained from Dr Weiguo Zhu [63]. Myogenin-Luc, MCK-Luc, MyHC-Luc and Tnni2-Luc were used as described [26, 64]. Renilla luciferase reporter plasmid was obtained from Promega and used according to the manufacturer's instructions (E1960, Promega, Madison, WI, USA). To construct Malat1 luciferase reporter plasmids, a 45 bp fragment encompassing WT mmu-miR-181a$5 \mathrm{p}$-binding site $\left(5^{\prime}\right.$-cAaagacetgtagagctgttgaatgtttgcagctggca-3') or mutated binding site (5'-cAaagacctgtagagctgcetgcgac ttgcagctggca-3') was cloned into pMIR-report vector (AM5795, Life Technologies, Carlsbad, CA, USA) between Spel and Sacl sites. To construct Malat1 shRNAi viral vector, a $61 \mathrm{bp}$ fragment (5'-gATCCggTgAATgAgTgATAAgTAATTCAAgAg ATTACTTATCACTCATTCACCTTTTTTg-3') harboring the target sequences was cloned into pSIREN RetroQ vector (631526, Clontech, Mountain View, CA, USA).

\section{Transfection and infection}

Transient transfection of siRNA oligos or plasmids was conducted with Lipofectamine 2000 following the instructions from the manufacturer (11668-019, Invitrogen). For the transfection of in vitro-transcribed RNA, RNA was heated at $70{ }^{\circ} \mathrm{C}$ for $10 \mathrm{~min}$. Lipofectamine 2000 was added directly to the RNA and then mixed by vortex. DMEM medium without serum or antibiotics was then added to the mixture and mixed by vortex. The final mixture was added to cells dropwise. For dual luciferase reporter assay, the firefly luciferase reporter plasmids were transfected to cells together with Renilla luciferase reporter plasmid, which worked as transfection efficiency control. To generate $\mathrm{C} 2 \mathrm{C} 12$ cells with stable knocking down of Malat1,

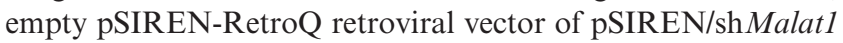
along with the packaging plasmid were transfected into HEK293T cells. Forty-eight hours after transfection, culture supernatants were collected and used for infecting C2C12 cells, which were later selected by $5 \mu \mathrm{g} \mathrm{ml}^{-1}$ puromycin.

\section{Oligonucleotides}

Precursor miRNA oligos were obtained from Life Technologies. siRNA oligos against mouse Malat1 and Ago2 were 
obtained from Ribobio Technologies (Guangzhou, China). Anti-miR-NC and miR-181a were obtained from Ribobio and transfected at a final concentration of 100 nM. siAgo 2 oligos or miRNA oligos $(50 \mathrm{nM})$ were used for transient transfections. Malat1 siRNAs were transfected at a final concentration of $150 \mathrm{~nm}$. The sequences of oligonucleotides used for siRNA knockdown, RT-PCR and ChIP-PCR were provided in the Supplementary Table S3.

\section{Quantitative real-time PCR}

Total RNAs were extracted using TRIzol reagent (Invitrogen). Expression of mRNA was performed with SYBR Green Master Mix (4368708, Applied Biosystem, Foster City, CA, USA) as previously described using GAPDH for normalization $[26,64]$. Expression of mature miRNAs was determined using the miRNA-specific TaqMan microRNA assay kit (Applied Biosystem) on an ABI PRISM 7900HT Sequence Detection System (Applied Biosystem). U6 was used for normalization.

\section{Northern blotting assay}

An amount $10 \mu \mathrm{g}$ of total RNAs were loaded in each well of $1.2 \%$ formaldehyde-denaturing agarose gel and after electrophoresis transferred to a charged nylon membrane overnight. After the transfer RNA was crosslinked using a UV crosslinker (Stratagene, San Diego, CA, USA) and pre-hybridized with ULTRAhyb hybridization buffer (AM8670, Applied Biosystems). Probes against mouse Malat1 transcript were amplified with the following PCR primers (forward 5'-GTTACCA GCCCAAACCTCAA-3'; reverse 5'-CTACATTCCCACCCA GCACT-3') and labeled with $[\alpha-32 \mathrm{P}] \mathrm{dCTP}$ with RadPrime DNA labeling system (Invitrogen). Probes were boiled and hybridized with membrane overnight at $42{ }^{\circ} \mathrm{C}$. After hybridization, the membrane was washed twice with $2 \times \mathrm{SSC}$ buffer and $0.1 \%$ SDS at room temperature and once with $0.1 \times \mathrm{SSC}$ and $0.1 \% \mathrm{SDS}$ at $55^{\circ} \mathrm{C}$ for $30 \mathrm{~min}$.

\section{Fluorescence in situ hybridization}

In situ hybridization was performed following the protocol from Dr KV Prasanth [11]. Probes were first amplified with PCR using Malat1 expression plasmid as template. PCR products were then purified, nick-translated and labeled with Green d-UTP (Abbott, Chicago, IL, USA) using a nick translation kit (Abbott). Cells were fixed with 2-4\% freshly prepared paraformaldehyde (in $1 \times \mathrm{PBS}$ ) $\mathrm{pH} 7.2$ for $15 \mathrm{~min}$ at RT. The cells were permeabilized with $0.2-0.5 \%$ Triton X-100, 2 mM VRC (NEBiolabs, Ipswich, MA, USA) on ice for 5-10 min and then washed two times in $2 \times \mathrm{SSC}$ for $10 \mathrm{~min}$. In all, $200 \mu \mathrm{g}$ or more of the probe and yeast tRNA (Sigma; $20 \mu \mathrm{g}$ ) were lyophilized and redissolved in $10 \mu$ deionized formamide (Ambion, Carlsbad, CA, USA), and denatured at $75-100^{\circ} \mathrm{C}$ for $10 \mathrm{~min}$ and immediately chilled in ice for $3-5 \mathrm{~min}$. In all, $10 \mu \mathrm{l}$ of hybridization buffer was added to each reaction to make a final hybridization cocktail of $20 \mu \mathrm{l}$ per coverslip. Coverslips were incubated at $37^{\circ} \mathrm{C}$ overnight in a humidified chamber. After the hybridization, coverslips were washed in $2 \times \mathrm{SSC}, 50 \%$ formamide $\left(\mathrm{pH} \mathrm{7.2)} \mathrm{for} 3 \times 5 \mathrm{~min}\right.$ at $42^{\circ} \mathrm{C}, 2 \times \mathrm{SSC}(\mathrm{pH} 7.2)$ for $3 \times 5$ min at $42^{\circ} \mathrm{C}, 1 \times \mathrm{SSC}(\mathrm{pH} 7.2)$ for $3 \times 5 \mathrm{~min}$ at $42^{\circ} \mathrm{C}$ and $4 \times \mathrm{SSC}$ for $2 \times 10 \mathrm{~min}$ at RT. Coverslips were mounted with
ProLong Gold Antifade Reagent with DAPI (Invitrogen). Pictures were captured in Kahlman frame, giving an average of two scans using the Olympus microscope FV1000 and the accompanying software FV10-ASW (version 01.07.02.02, Olympus, Tokyo, Japan).

\section{$R N A$ immunoprecipitation}

RIP assay was conducted as previously described [65]. Two micrograms of antibodies against Suv39h1 (Millipore, Darmstadt, USA), Ago2 (Abcam, Cambridge, MA, USA), Dicer (Santa Cruz Biotechnology, Santa Cruz, CA, USA), Trbp (Abcam), Tnrc6a (Santa Cruz Biotechnology) or isotype IgG (Santa Cruz Biotechnology) were used in this assay. Pulled down RNA were resuspended in $20 \mu \mathrm{l}$ of RNase-free water, and cDNAs were obtained from reverse transcription. qRT-PCR was performed with cDNA by using SYBR Green Master Mix (Applied Biosystems). Relative enrichment is calculated as the amount of amplified DNA normalized to values obtained after normal IgG immunoprecipitation.

\section{$R N A$-seq and data analysis}

For library construction, we used a protocol as described before [66]. The purified library products were evaluated using a Bioanalyzer (Agilent) and SYBR qPCR and sequenced on an Illumina Hi-seq 2000 sequencer (pair-end with $50 \mathrm{bp}$ ). Sequenced fragments were mapped to reference mouse genome using TopHat. Cufflinks was then used to estimate transcript abundances of RNA-Seq experiments. Abundances were reported in Fragments Per Kilobase per Million, which is conceptually analogous to the reads per kilobase per million reads mapped used for single-end RNA-seq. Differentially expressed genes were identified using Cuffdiff with default parameters. A false discovery rate-adjusted $P$-value (or $q$-value) of 0.05 was used as the cutoff in the analysis. [67].

\section{Immunoblotting, immunostaining and immunohistochemistry}

For western blotting, total cell proteins were prepared and used as previously described [64]. The following dilutions were used for each antibody: Myogenin (Santa Cruz Biotechnology; 1:2 000), YY1 (Santa Cruz Biotechnology; 1:2 000), Troponin (Sigma; 1:2 000), MyHC (Sigma; 1:2 000), $\alpha$-Actin (Sigma; 1:2 000), $\alpha$-Tubulin (Sigma; 1:5000), MyoD (Santa Cruz Biotechnology; 1:2 000), Suv39h1 (Millipore, 1:2 000), HDAC1 (Abcam, 1:2 000), HP1 $\beta$ (Abcam, 1:2 000), Ago2 (Abcam, 1:2 000), Dicer (Santa Cruz Biotechnology, 1:2000), Trbp (Abcam, 1:5 000), Tnrc6a (Santa Cruz Biotechnology, 1:2 000) and GAPDH (Santa Cruz Biotechnology; 1:5 000). Immunofluorescence on cultured cells was performed using the following antibodies: MyHC (Sigma; 1:350), Myogenin (Santa Cruz Biotechnology, 1:400) and Srsf2 (Abcam; 1:800). Immunofluorescence staining on frozen muscle sections was performed using the following antibodies: Laminin (Santa Cruz Biotechnology, 1:400), Myogenin (Santa Cruz Biotechnology; 1:400) and eMyHC (Leica, 1:300). Immunofluorescence on single myofibers was performed using the following antibodies: Pax7 (Developmental Studies Hybridoma Bank; 1:200) and Myogenin (Santa Cruz Biotechnology; 1:200). Hematoxylin and 
eosin (H\&E) staining on frozen muscle sections was performed as previously described [68]. All fluorescent images were captured with a fluorescence microscope (Leica).

\section{Chromatin immunoprecipitation}

ChIP assay was performed as previously described [29]. Five micrograms of antibodies against Suv39h1 (Millipore), Hp1 $\beta$ (Abcam), HDAC1 (Abcam), trimethyl-histone H3-K9 (Abcam), MyoD (Santa Cruz Biotechnology), Set7 (Abcam) or isotype IgG (Santa Cruz Biotechnology), which was applied as a negative control, were used in the assay. Pulled down DNAs were resuspended in $20 \mu \mathrm{l}$ of water. qRT-PCR was performed with $1 \mu$ of immunoprecipitated material by using SYBR Green Master Mix (Applied Biosystems). Relative enrichment is calculated as the amount of amplified DNA normalized to input and relative to values obtained after normal $\mathrm{IgG}$ immunoprecipitation.

\section{Chromatin isolation by $R N A$ purification}

ChIRP was conducted as previously described [45]. Biotinlabeled antisense oligos (20 nucleotides long) were designed and divided into odd and even pools. Briefly, C2C12 cells were harvested and crosslinked with $1 \%$ glutaraldehyde. An amount of $100 \mathrm{mg}$ of cell pellet were used for one pull down. Cells were sonicated at $4{ }^{\circ} \mathrm{C}$ for $3 \mathrm{~h}$ to shear the DNA to $100-500 \mathrm{bp}$. Pooled odd and even probes were hybridized with sonicated chromatins and then pulled down by C-1 streptavidin beads (65001, Invitrogen). Pulled down RNA and DNA were isolated. Isolated RNA was reverse-transcribed using both oligodT and random hexamer, and the cDNA was used to perform qRT-PCR to check the pull-down efficiency. qRT-PCR was conducted with retrieved DNA using the primers for ChIP to check the enrichment of Malat1 at certain DNA loci.

\section{RNA EMSA}

EMSA for RNA was performed as previously described [69]. GST alone or GST-fused proteins were expressed in DE3 bacteria and induced by IPTG for $4 \mathrm{~h}$ at $28^{\circ} \mathrm{C}$ and purified by glutathione-Sepharose 4B beads (GE Healthcare, Waukesha, WI, USA). In vitro-transcribed RNAs were heated to $90^{\circ} \mathrm{C}$ for 2 min with $10 \times$ RNA structure buffer $(100 \mathrm{~mm}$ Tris $\mathrm{pH} 7,1 \mathrm{M}$ $\mathrm{KCl}, 100 \mathrm{~mm} \mathrm{MgCl}_{2}$ ) and then put on ice for $2 \mathrm{~min}$ followed by incubation at $\mathrm{RT}$ for $20 \mathrm{~min}$. Reaction mixtures containing $50 \mathrm{~mm}$ Tris $\mathrm{HCl} \mathrm{pH} 7.0,150 \mathrm{~mm} \mathrm{NaCl}, 0.25 \mathrm{mg} \mathrm{ml}^{-1}$ tRNA, $0.25 \mathrm{mg} \mathrm{ml}^{-1}$ bovine serum albumin, RNA and protein were mixed and incubated at $37^{\circ} \mathrm{C}$ for $10 \mathrm{~min}$. Following incubation, mixtures were immediately loaded to $0.8 \%$ non-denaturing agarose gels with loading buffer in $0.5 \times \mathrm{TAE}$.

\section{Animal studies}

Malat1 $^{-1-}$ KO mice were obtained from Dr Shinichi Nakagawa, and the genotyping of the mice was conducted following the procedures previously described in their paper [40]. C57 BL/10 ScSn-Dmd ${ }^{\text {mdx }}$ mice were obtained from the Jackson Laboratory (Bar Harbor, ME, USA). Malat $^{-1-}$ male mice were crossed with homozygous Dmd ${ }^{\mathrm{mdx} / \mathrm{mdx}}$ female mice to generate Malat1 $^{+/-}$; Dmd ${ }^{\mathrm{mdx} / \mathrm{y}}$ male mice. Malat $^{+/-}$; $\mathrm{Dmd}^{\mathrm{mdx} / \mathrm{y}}$ male mice were then bred with Dmd ${ }^{\mathrm{mdx} / \mathrm{mdx}}$ female mice to obtain Malat $^{+/-}$;
Dmd $^{\text {mdx/mdx }}$ female mice. Malat $1^{+/-}$; Dmd ${ }^{\mathrm{mdx} / \mathrm{y}}$ male mice and Malat $^{+/-}$; Dmd ${ }^{\text {mdx/mdx }}$ female mice were then crossed to generate Malat1 $^{-1-} ; \mathrm{Dmd}^{\mathrm{mdx} / \mathrm{y}}$ and Malat1 $^{-1-} ; \mathrm{Dmd}^{\mathrm{mdx} / \mathrm{mdx}}$ mice, which were referred as $\mathrm{mdx} /$ Malat $1-\mathrm{KO}$ mice. Littermate $\mathrm{mdx} /$ Malat1WT mice were used as controls. Mice were housed in the animal facilities of The Chinese University of Hong Kong (CUHK) under conventional conditions with constant temperature and humidity, and were fed a standard diet. Animal experimentation was approved by the CUHK Animal Experimentation Ethics Committee (Ref No. 14-059-GRF). Postnatal muscles were obtained from C57/BL6 mice for RNA extraction followed by RT-PCR analysis. For Cardiotoxin studies, $\sim 8$-week-old mice were injected with $50 \mu \mathrm{l}$ of $10 \mu \mathrm{g} \mathrm{ml}^{-1}$ Cardiotoxin (Latoxan, France) solution into each tibialis anterior muscle. Muscles were harvested at designated time points for further analysis.

\section{Statistical test}

The statistical significance was assessed by the Student's $t$-test. $* P<0.05, * * P<0.01$ and $* * * P<0.001$.

\section{Conflict of Interest}

The authors declare no conflict of interest.

\section{Acknowledgements}

The work described in this paper was substantially supported by a General Research Funds (GRF) from the Research Grants Council (RGC) of the Hong Kong Special Administrative Region, China to H.W. (Project Number: 14116014) and several other GRF projects (14133016, 14100415 for H.W. and 14113514, 14102315 for H.S.), a Collaborative Research Fund (CRF) (Project Code: C-6015-14G), grants from the Ministry of Science and Technology of China (2014CB964700), the National Natural Science Foundation of China (31371513 and 31671537). We thank Professor Dennis YM Lo and Professor Rossa WK Chiu for granting us the access to GAIIx sequencer and their generous support throughout the course of this study. We thank Professor Jacky Ngo for sharing the Srsf2 antibody. We are also grateful to Professor Tom Cheung for sharing several protocols.

\section{Author contributions}

XC designed and performed most experiments, analyzed data, interpreted results and wrote the manuscript. LH, YZ, YL, SZ, KS, KS, FC, LZ, LL and LW performed individual experiments and analyzed data. XZ, XB and ME provided support and suggestions for ChIRP assay. SN provided the Malat1 knockout mice. KVP provided support and suggestions for fluorescence in situ hybridization. ZW provided support and suggestions for Gal4 luciferase reporter assay. HS and HW conceived the project, designed experiments, interpreted results and wrote the manuscript. 


\section{References}

1 Fatica A, Bozzoni I. Long non-coding RNAs: new players in cell differentiation and development. Nat Rev Genet 2014; 15: 7-21.

2 Batista PJ, Chang HY. Long noncoding RNAs: cellular address codes in development and disease. Cell 2013; 152: 1298-1307.

3 Lee JT. Epigenetic regulation by long noncoding RNAs. Science 2012; 338: 1435-1439.

4 Moran VA, Perera RJ, Khalil AM. Emerging functional and mechanistic paradigms of mammalian long noncoding RNAs. Nucleic Acids Res 2012; 40: 6391-6400.

5 Rinn JL, Chang HY. Genome regulation by long noncoding RNAs. Annu Rev Biochem 2012; 81: 145-166.

6 Wang KC, Chang HY. Molecular mechanisms of long noncoding RNAs. Mol Cell 2011; 43: 904-914.

7 Ji P, Diederichs S, Wang W et al. MALAT-1, a novel noncoding RNA, and thymosin beta4 predict metastasis and survival in early-stage non-small cell lung cancer. Oncogene 2003; 22: 8031-8041.

8 Gutschner T, Diederichs S. The hallmarks of cancer: a long non-coding RNA point of view. RNA Biol 2012; 9: 703-719.

9 Gutschner T, Hammerle M, Diederichs S. MALAT1 -- a paradigm for long noncoding RNA function in cancer. J Mol Med 2013; 91: 791-801.

10 Hutchinson JN, Ensminger AW, Clemson CM, Lynch CR, Lawrence JB, Chess A. A screen for nuclear transcripts identifies two linked noncoding RNAs associated with SC35 splicing domains. BMC Genomics 2007; 8: 39.

11 Tripathi V, Ellis JD, Shen Z et al. The nuclear-retained noncoding RNA MALAT1 regulates alternative splicing by modulating SR splicing factor phosphorylation. Mol Cell 2010; 39: 925-938.

12 Bernard D, Prasanth KV, Tripathi V et al. A long nuclearretained non-coding RNA regulates synaptogenesis by modulating gene expression. EMBO J 2010; 29: 3082-3093.

13 Yang L, Lin C, Liu W et al. ncRNA- and Pc2 methylationdependent gene relocation between nuclear structures mediates gene activation programs. Cell 2011; 147: 773-788.

14 Yoon JH, Abdelmohsen K, Gorospe M. Functional interactions among microRNAs and long noncoding RNAs. Semin Cell Dev Biol 2014; 34: 9-14.

15 Cesana M, Cacchiarelli D, Legnini I et al. A long noncoding RNA controls muscle differentiation by functioning as a competing endogenous RNA. Cell 2011; 147: 358-369.

16 Hirata H, Hinoda Y, Shahryari V et al. Long noncoding RNA MALAT1 promotes aggressive renal cell Carcinoma through Ezh2 and interacts with miR-205. Cancer Res 2015; 75: 1322-1331.

17 Xiao H, Tang K, Liu P et al. LncRNA MALAT1 functions as a competing endogenous RNA to regulate ZEB2 expression by sponging miR-200s in clear cell kidney carcinoma. Oncotarget 2015; 6: 38005-38015.
18 Lu L, Luo F, Liu Y et al. Posttranscriptional silencing of the lncRNA MALAT1 by miR-217 inhibits the epithelialmesenchymal transition via enhancer of zeste homolog 2 in the malignant transformation of HBE cells induced by cigarette smoke extract. Toxicol Appl Pharmacol 2015; 289: 276-285.

19 Rudnicki MA, Le Grand F, McKinnell I, Kuang S. The molecular regulation of muscle stem cell function. Cold Spring Harb Symp Quant Biol 2008; 73: 323-331.

20 Brack AS, Rando TA. Tissue-specific stem cells: lessons from the skeletal muscle satellite cell. Cell Stem Cell 2012; 10: $504-514$.

21 Braun T, Gautel M. Transcriptional mechanisms regulating skeletal muscle differentiation, growth and homeostasis. Nat Rev Mol Cell Biol 2011; 12: 349-361.

22 Lu L, Zhou L, Chen EZ et al. A Novel YY1-miR-1 regulatory circuit in skeletal myogenesis revealed by genome-wide prediction of YY1-miRNA network. PLoS ONE 2012; 7: e27596.

23 Wang L, Zhou L, Jiang P et al. Loss of miR-29 in myoblasts contributes to dystrophic muscle pathogenesis. Mol Ther 2012; 20: 1222-1233.

24 Zhou L, Wang L, Lu L, Jiang P, Sun H, Wang H. A novel target of microRNA-29, Ring1 and YY1-binding protein (Rybp), negatively regulates skeletal myogenesis. J Biol Chem 2012; 287: 25255-25265.

25 Zhou L, Wang L, Lu L, Jiang P, Sun H, Wang H. Inhibition of miR-29 by TGF-beta-Smad3 signaling through dual mechanisms promotes transdifferentiation of mouse myoblasts into myofibroblasts. PLoS ONE 2012; 7: e33766.

26 Wang H, Garzon R, Sun H et al. NF-kappaB-YY1-miR-29 regulatory circuitry in skeletal myogenesis and rhabdomyosarcoma. Cancer Cell 2008; 14: 369-381.

27 Sartorelli V, Juan AH. Sculpting chromatin beyond the double helix: epigenetic control of skeletal myogenesis. Curr Top Dev Biol 2012; 96: 57-83.

28 Saccone V, Puri PL. Epigenetic regulation of skeletal myogenesis. Organogenesis 2010; 6: 48-53.

29 Lu L, Sun K, Chen X et al. Genome-wide survey by ChIP-seq reveals YY1 regulation of lincRNAs in skeletal myogenesis. EMBO J 2013; 32: 2575-2588.

30 Wang L, Zhao Y, Bao X et al. LncRNA Dum interacts with Dnmts to regulate Dppa2 expression during myogenic differentiation and muscle regeneration. Cell Res 2015; 25 : 335-350.

31 Watts R, Johnsen VL, Shearer J, Hittel DS. Myostatininduced inhibition of the long noncoding RNA Malat1 is associated with decreased myogenesis. Am J Physiol Cell Physiol 2013; 304: C995-1001.

32 Han X, Yang F, Cao H, Liang Z. Malat1 regulates serum response factor through miR-133 as a competing endogenous RNA in myogenesis. FASEB J 2015; 29: 3054-3064.

33 Aziz A, Liu QC, Dilworth FJ. Regulating a master regulator: establishing tissue-specific gene expression in skeletal muscle. Epigenetics 2010; 5: 691-695.

34 Cao Y, Yao Z, Sarkar D et al. Genome-wide MyoD binding in skeletal muscle cells: a potential for broad cellular reprogramming. Dev Cell 2010; 18: 662-674. 
35 Mal AK. Histone methyltransferase Suv39h1 represses MyoD-stimulated myogenic differentiation. EMBO J 2006; 25: 3323-3334.

36 Tao Y, Neppl RL, Huang ZP et al. The histone methyltransferase Set7/9 promotes myoblast differentiation and myofibril assembly. J Cell Biol 2011; 194: 551-565.

37 Yahi H, Fritsch L, Philipot O et al. Differential cooperation between heterochromatin protein HP1 isoforms and MyoD in myoblasts. J Biol Chem 2008; 283: 23692-23700.

38 Eissmann M, Gutschner T, Hammerle $\mathrm{M}$ et al. Loss of the abundant nuclear non-coding RNA MALAT1 is compatible with life and development. RNA Biol 2012; 9: 1076-1087.

39 Zhang B, Arun G, Mao YS et al. The lncRNA Malat1 is dispensable for mouse development but its transcription plays a cis-regulatory role in the adult. Cell Rep 2012; 2: 111-123.

40 Nakagawa S, Ip JY, Shioi G et al. Malat1 is not an essential component of nuclear speckles in mice. RNA 2012; 18: 1487-1499.

41 Trapnell C, Williams BA, Pertea G et al. Transcript assembly and quantification by RNA-Seq reveals unannotated transcripts and isoform switching during cell differentiation. Nat Biotechnol 2010; 28: 511-515.

42 Tripathi V, Song DY, Zong X et al. SRSF1 regulates the assembly of pre-mRNA processing factors in nuclear speckles. Mol Biol Cell 2012; 23: 3694-3706.

43 Castelo-Branco G, Amaral PP, Engstrom PG et al. The non-coding snRNA 7SK controls transcriptional termination, poising, and bidirectionality in embryonic stem cells. Genome Biol 2013; 14: R98.

44 Dillon SC, Zhang X, Trievel RC, Cheng X. The SET-domain protein superfamily: protein lysine methyltransferases. Genome Biol 2005; 6: 227.

45 Chu C, Quinn J, Chang HY. Chromatin isolation by RNA purification (ChIRP). J Vis Exp 2012; 25: pii: 3912.

46 Leung AK, Young AG, Bhutkar A et al. Genome-wide identification of Ago2 binding sites from mouse embryonic stem cells with and without mature microRNAs. Nat Struct Mol Biol 2011; 18: 237-244.

47 Naguibneva I, Ameyar-Zazoua M, Polesskaya A et al. The microRNA miR-181 targets the homeobox protein Hox-A11 during mammalian myoblast differentiation. Nat Cell Biol 2006; 8: 278-284.

48 Roberts TC. The microRNA biology of the mammalian nucleus. Mol Ther Nucleic Acids 2014; 3: e188.

49 Chendrimada TP, Gregory RI, Kumaraswamy E et al. TRBP recruits the Dicer complex to Ago2 for microRNA processing and gene silencing. Nature 2005; 436: $740-744$.

50 Nishi K, Nishi A, Nagasawa T, Ui-Tei K. Human TNRC6A is an Argonaute-navigator protein for microRNA-mediated gene silencing in the nucleus. $R N A$ 2013; 19: 17-35.

51 West JA, Davis CP, Sunwoo H et al. The long noncoding RNAs NEAT1 and MALAT1 bind active chromatin sites. Mol Cell 2014; 55: 791-802.

52 Wilusz JE, JnBaptiste CK, Lu LY, Kuhn CD, Joshua-Tor L, Sharp PA. A triple helix stabilizes the 3' ends of long noncoding RNAs that lack poly(A) tails. Genes Dev 2012; 26: 2392-2407.

53 Xu C, Yang M, Tian J, Wang X, Li Z. MALAT-1: a long non-coding RNA and its important 3 ' end functional motif in colorectal cancer metastasis. Int J Oncol 2011; 39: 169-175.

54 Krajewski WA, Nakamura T, Mazo A, Canaani E. A motif within SET-domain proteins binds single-stranded nucleic acids and transcribed and supercoiled DNAs and can interfere with assembly of nucleosomes. Mol Cell Biol 2005; 25: 1891-1899.

55 Krajewski WA, Reese JC. SET domains of histone methyltransferases recognize ISWI-remodeled nucleosomal species. Mol Cell Biol 2010; 30: 552-564.

56 Tripathi V, Shen Z, Chakraborty A et al. Long noncoding RNA MALAT1 controls cell cycle progression by regulating the expression of oncogenic transcription factor B-MYB. PLoS Genet 2013; 9: e1003368.

57 Arun G, Diermeier S, Akerman M et al. Differentiation of mammary tumors and reduction in metastasis upon Malat1 lncRNA loss. Genes Dev 2016; 30: 34-51.

58 Michalik KM, You X, Manavski Y et al. Long noncoding RNA MALAT1 regulates endothelial cell function and vessel growth. Circ Res 2014; 114: 1389-1397.

59 Nakagawa S, Shimada M, Yanaka K et al. The lncRNA Neatl is required for corpus luteum formation and the establishment of pregnancy in a subpopulation of mice. Development 2014; 141: 4618-4627.

60 Rocheteau P, Gayraud-Morel B, Siegl-Cachedenier I, Blasco MA, Tajbakhsh S. A subpopulation of adult skeletal muscle stem cells retains all template DNA strands after cell division. Cell 2012; 148: 112-125.

61 Cheung TH, Quach NL, Charville GW et al. Maintenance of muscle stem-cell quiescence by microRNA-489. Nature 2012; 482: 524-528.

62 Cabianca DS, Casa V, Bodega B et al. A long ncRNA links copy number variation to a polycomb/trithorax epigenetic switch in FSHD muscular dystrophy. Cell 2012; 149: 819-831.

63 Wang D, Zhou J, Liu X et al. Methylation of SUV39H1 by $\mathrm{SET} 7 / 9$ results in heterochromatin relaxation and genome instability. Proc Natl Acad Sci USA 2013; 110: 5516-5521.

64 Wang H, Hertlein E, Bakkar $\mathrm{N}$ et al. NF-kappaB regulation of YY1 inhibits skeletal myogenesis through transcriptional silencing of myofibrillar genes. Mol Cell Biol 2007; 27: 43744387.

65 Tsai MC, Manor O, Wan Y et al. Long noncoding RNA as modular scaffold of histone modification complexes. Science 2010; 329: 689-693.

66 Zhou L, Wang L, Lu L, Jiang P, Sun H, Wang H. Inhibition of miR-29 by TGF-beta-Smad3 signaling through dual mechanisms promotes transdifferentiation of mouse myoblasts into myofibroblasts. PLoS ONE 7: e33766.

67 Zhang ZH, Jhaveri DJ, Marshall VM et al. A comparative study of techniques for differential expression analysis on RNA-Seq data. PLoS ONE 2014; 9: e103207. 
68 Diao Y, Guo X, Li Y et al. Pax3/7BP is a Pax7- and Pax3-binding protein that regulates the proliferation of muscle precursor cells by an epigenetic mechanism. Cell Stem Cell 11: 231-241.

69 Li Y, Jiang Z, Chen H, Ma WJ. A modified quantitative EMSA and its application in the study of RNA--protein interactions. J Biochem Biophys Methods 2004; 60: 85-96.

(Supplementary Information is linked to the online version of the paper on the Cell Discovery website.) (c) (i) This work is licensed under a Creative Commons Attribution 4.0 International License. The images or other third party material in this article are included in the article's Creative Commons license, unless indicated otherwise in the credit line; if the material is not included under the Creative Commons license, users will need to obtain permission from the license holder to reproduce the material. To view a copy of this license, visit http://creativecommons.org/licenses/by/4.0/

(C) The Author(s) 2017 